\title{
Lewis Base Activation of Lewis Acids. Vinylogous Aldol Reactions
}

\author{
Scott E. Denmark* and Gregory L. Beutner \\ Roger Adams Laboratory, Department of Chemistry, University of Illinois \\ Urbana, Illinois 61801
}

\section{SUPPORTING INFORMATION}

\section{General Experimental}

All reactions were performed in oven-dried $\left(140{ }^{\circ} \mathrm{C}\right)$ or flame-dried glassware under an atmosphere of dry $\mathrm{N}_{2}$. The following reaction solvents were distilled from the indicated drying agents: dichloromethane $\left(\mathrm{P}_{2} \mathrm{O}_{5}\right)$, benzene (Fisher ACS grade), hexanes (Fisher ACS grade), pentane (Fisher, ACS grade), acetonitrile (Fisher, ACS grade), tetrahydrofuran (Na, benzophenone) and diethyl ether ( $\mathrm{Na}$, benzophenone). Solvents for chromatography and filtration were technical grade and distilled from the indicated drying agents: hexane $\left(\mathrm{CaCl}_{2}\right)$, ethyl acetate $\left(\mathrm{K}_{2} \mathrm{CO}_{3}\right)$, dichloromethane $\left(\mathrm{CaCl}_{2}\right)$. Column chromatography was performed using EM Science 230-400-mesh silica gel. Benzaldehyde (1a), (E)-cinnamaldehyde (1b), hydrocinnamaldehyde (1c) and 2,2,6-Trimethyl-1,3-dioxin-4-one (Lancaster) were freshly distilled before use. 1-(tert-butyldimethylsilyloxy)-1-methoxyethene (1) (Aldrich), methyl [(1Methoxy-2-methyl-1-propenyl)oxy]trimethylsilane (12) and tert-butyl dimethylsilyl chloride (TBSCl, Aldrich), ethyl 3,3-dimethyl acrylate (Aldrich), methyl tiglate (Lancaster), ethyl crotonate (Aldrich) and (E)-2-pentenoic acid (Lancaster) were used without further purification. Silicon tetrachloride (Gelest) was heated at reflux for $24 \mathrm{~h}$ and then distilled prior to use. HMPA was distilled and then stored over $3 \AA$ molecular sieves prior to use. Diisopropylamine, diisopropylethylamine (DIPEA) and pyridine were freshly distilled from $\mathrm{CaH}_{2}$ prior to use. All reaction temperatures correspond to bath temperatures unless otherwise noted. Internal temperatures were measured by Teflon-coated thermocouples during optimization studies.

${ }^{1} \mathrm{H}$ NMR, ${ }^{13} \mathrm{C}$ NMR and low temperature spectra were recorded on a Varian Unity 400 $\left(400 \mathrm{MHz},{ }^{1} \mathrm{H} ; 100 \mathrm{MHz},{ }^{13} \mathrm{C}\right)$, Unity $500\left(500 \mathrm{MHz},{ }^{1} \mathrm{H} ; 126 \mathrm{MHz},{ }^{13} \mathrm{C}\right)$ spectrometers. Spectra were referenced to residual chloroform $\left(7.26 \mathrm{ppm},{ }^{1} \mathrm{H}, 77.23 \mathrm{ppm},{ }^{13} \mathrm{C}\right)$. Chemical shifts are reported in ppm, multiplicities are indicated by s (singlet), $d$ (doublet), $t$ (triplet), q (quartet), $p$ (pentet) h (hextet) $\mathrm{m}$ (multiplet) and br (broad). Coupling constants, $J$, are reported in Hertz. Mass spectroscopy was performed by the University of Illinois Mass Spectrometer Center. EI 
and CI mass spectra were performed on a 70-VSE spectrometer. Data are reported in the form of $(\mathrm{m} / \mathrm{z})$. Infrared spectra (IR) were recorded on a Mattson Galaxy 5020 spectrophotometer in $\mathrm{NaCl}$ cells. Peaks are reported in $\mathrm{cm}^{-1}$ with indicated relative intensities: s (strong, 67-100\%); $\mathrm{m}$ (medium, 34-66\%); w (weak, 0-33\%). Elemental analyses were performed by the University of Illinois Microanalytical Service Laboratory. Melting points ( $\mathrm{mp}$ ) were determined on a ThomasHoover capillary melting point apparatus in sealed tubes and are corrected. Kugelrohr distillations were performed on a Büchi GKR-50 Kugelrohr and temperatures reported are air bath temperatures. Analytical thin-layer chromatography was performed on Merck silica gel plates with QF-254 indicator. Visualization was accomplished with UV(254), potassium permanganate $\left(\mathrm{KMnO}_{4}\right)$ and p-anisaldehyde.

Optical rotation data was obtained on a JASCO DIP-360 digital polarimeter and are reported as follows: concentration $(\mathrm{c}=\mathrm{g} / 100 \mathrm{~mL})$, and solvent. Chrial stationary phase, supercritical fluid chromatography (CSP-SFC) was performed on a Berger Instruments packedcolumn SFC with built-in photometric detector $(220 \mathrm{~nm})$ using Daicel Chiralpak OD, OJ, AD, AS, OB columns and a Regis Whelk-O1 column. All retenion times $\left(t_{R}\right)$ are reported in min.

Ozonolyses were performed with a Welsbach Model T-816 Ozonator set at $55 \mathrm{~W} / 90 \mathrm{~V}$ with a 1 slpm flow rate.

\section{Literature Preparations.}

$(R, R)-N, N^{\prime}$-Bis[4,5-dihydro-3,5-dimethyl-4-(3H-dinaphtho[2,1-d:1',2'-f][1,3,2]-2-oxodiazaphosphepino)]-N,N'-dimethyl-1,5-pentanediamine $\quad((R, R)-5)^{1} \quad$ and $\quad(E)$-[(1-methoxy)propenyloxy)-tert-butyldimethylsilane $(\mathbf{1 0})^{2}$ were prepared according to established procedures. 


\section{Experimental Procedures.}

\section{General Procedure 1. Enolization of Alkenoates to Silyl Dienol Ethers.}

Preparation of $(Z)$-tert-Butyl-(1-ethoxy-1,3-butadienyloxy)dimethylsilane ((Z)-3a)

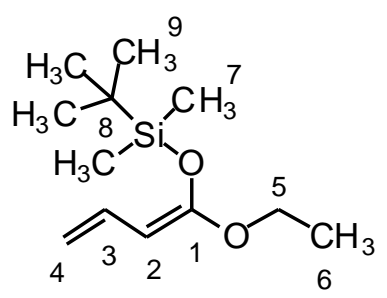

3a

To a flame-dried, $100 \mathrm{~mL}$, 3-neck round-bottom flask was added $1.54 \mathrm{~mL}$ of diisopropylamine (11 mmol, 1.1 equiv) and $11 \mathrm{~mL}$ of THF. The solution was cooled to $0{ }^{\circ} \mathrm{C}$ in an ice-water bath and then $7.1 \mathrm{~mL}$ of $n$-BuLi (1.55 $\mathrm{M}$ in hexane, $11 \mathrm{mmol}, 1.1$ equiv) was added slowly over $5 \mathrm{~min}$. The resulting solution was stirred for $30 \mathrm{~min}$ prior to cooling to $-78{ }^{\circ} \mathrm{C}$ in a dry ice/isopropanol bath. To this solution was added $2.1 \mathrm{~mL}$ of HMPA (12 mmol, 1.2 equiv). The solution was stirred for $30 \mathrm{~min}$ at $-78{ }^{\circ} \mathrm{C}$ and a white precipitate formed. Then, $1.24 \mathrm{~mL}$ of ethyl crotonate (10 mmol, 1.0 equiv.) was added slowly over $5 \mathrm{~min}$. The resulting solution was stirred for $30 \mathrm{~min}$ at $-78{ }^{\circ} \mathrm{C}$ prior to slow addition of $1.65 \mathrm{~g}$ of $\mathrm{TBSCl}$ (11.6 mmol, 1.2 equiv) in $6 \mathrm{~mL}$ of THF via cannula. The dry ice/isopropanol bath was then removed and the solution was allowed to stir for $1 \mathrm{~h}$ at room temperature. The yellow solution was diluted with cold pentane $(50 \mathrm{~mL})$ and washed with cold $\mathrm{H}_{2} \mathrm{O}(3 \times 50 \mathrm{~mL})$. The organic layer was dried over $\mathrm{Na}_{2} \mathrm{SO}_{4}(10$ $\mathrm{g}$ ), filtered through a pad of Celite and the filtrate was concentrated in vacuo. The residue was purified by short path distillation to yield $1.63 \mathrm{~g}(71 \%, 86 / 14 \mathrm{Z} / E)$ of 3a as a colorless oil.

Data for (Z)-3a:

bp: $\quad 70{ }^{\circ} \mathrm{C}(1 \mathrm{~mm} \mathrm{Hg})$

${ }^{1} \mathrm{H}$ NMR: $\quad\left(500 \mathrm{MHz}, \mathrm{CDCl}_{3}\right)$

$6.53(\mathrm{dt}, J=17.1,10.5,1 \mathrm{H}, \mathrm{HC}(3)), 4.81$ (dd, $J=17.5,1.9,1 \mathrm{H}, \mathrm{HC}(4)), 4.57$ $(\mathrm{dd}, J=10.7,1.5,1 \mathrm{H}, \mathrm{HC}(4)), 4.43$ (d, $J=10.5,1 \mathrm{H}, \mathrm{HC}(2)), 3.79$ (q, $J=7.1,2$ $\mathrm{H}, \mathrm{HC}(5)), 1.30$ (t, $\left.J=7.1,3 \mathrm{H}, \mathrm{H}_{3} \mathrm{C}(6)\right), 0.95$ (s, $\left.9 \mathrm{H}, \mathrm{H}_{3} \mathrm{C}(9)\right), 0.19$ (s, $6 \mathrm{H}$, $\left.\mathrm{H}_{3} \mathrm{C}(7)\right)$ 
NOE ${ }^{1} \mathrm{H}$ NMR: $\left(500 \mathrm{MHz}, \mathrm{CDCl}_{3}\right)$

Irradiation at $3.79 \mathrm{ppm}(\mathrm{HC}(5))$ enhanced signal at $4.43 \mathrm{ppm}(\mathrm{HC}(2))$ in major isomer

${ }^{13} \mathrm{C} \mathrm{NMR}: \quad\left(100 \mathrm{MHz}, \mathrm{CDCl}_{3}\right)$

$157.73(\mathrm{C}(1)), 132.58(\mathrm{C}(3)), 106.26(\mathrm{C}(4)), 80.52$ (C(5)), $63.26(\mathrm{C}(2)), 25.69$

(C(9)), 25.52 (C(6)), $14.35(\mathrm{C}(8)),-4.15(\mathrm{C}(7))$

IR: (neat)

3085 (w), 2958 (m), 2933 (m), 2888 (m), 2859 (m), 1648 (s), 1602 (w), 1473 (w), 1425 (m), 1369 (m), 1334 (m), 1255 (m), 1214 (s), 1176 (m), 1079 (m), 1025 (m)

MS: $\quad(\mathrm{CI}, 70 \mathrm{eV})$

$229\left(\mathrm{M}^{+}, 27\right), 205$ (26), 187 (36), 171 (36), 147 (30), 115 (52), 75 (100)

HRMS: calcd for $\mathrm{C}_{12} \mathrm{H}_{24} \mathrm{O}_{2} \mathrm{Si}$ : 229.1623, found: 229.1249

tert-Butyl-(1-methoxy-2-methyl-1,3-butadienyloxy)dimethylsilane ((Z)-3b)

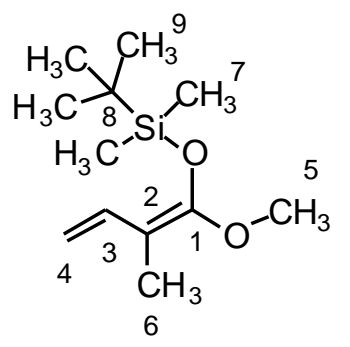

3b

Following General Procedure 1, $1.54 \mathrm{~mL}$ of diisopropylamine (11 mmol,1.1 equiv) was combined with $7.1 \mathrm{~mL}$ of $n-\mathrm{BuLi}$ (1.55 $\mathrm{M}$ in hexane, $11 \mathrm{mmol}, 1.1$ equiv), $2.1 \mathrm{~mL}$ of HMPA (12 mmol, 1.2 equiv), $1.2 \mathrm{~mL}$ of methyl tiglate (10 mmol, 1.0 equiv.) and $1.65 \mathrm{~g}$ of TBSCl (11.6 mmol, 1.2 equiv) to yield, after short path distillation, $1.48 \mathrm{~g}(65 \%, 58: 42 \mathrm{Z} / \mathrm{E})$ of $\mathbf{3 b}^{3}$ as a colorless oil. 
Data for $(Z)-3 \mathbf{b}$ :

bp: $\quad 66{ }^{\circ} \mathrm{C}(1 \mathrm{~mm} \mathrm{Hg})$

${ }^{1} \mathrm{H}$ NMR: $\quad\left(500 \mathrm{MHz}, \mathrm{CDCl}_{3}\right)$

$6.76(\mathrm{dd}, J=17.6,11.0,1 \mathrm{H}, \mathrm{HC}(3)), 4.85(\mathrm{dd}, J=17.6,1.5,1 \mathrm{H}, \mathrm{HC}(4)), 4.78$ $(\mathrm{dd}, J=10.5,1.5,1 \mathrm{H}, \mathrm{HC}(4)), 3.58$ (s, $\left.3 \mathrm{H} \mathrm{H}_{3} \mathrm{C}(5)\right), 1.63$ (s, $\left.3 \mathrm{H}, \mathrm{H}_{3} \mathrm{C}(6)\right), 0.97$

(s, $\left.9 \mathrm{H}, \mathrm{H}_{3} \mathrm{C}(9)\right), 0.18$ (s, $6 \mathrm{H}, \mathrm{H}_{3} \mathrm{C}(7)$ )

${ }^{13} \mathrm{C} \mathrm{NMR}: \quad\left(100 \mathrm{MHz}, \mathrm{CDCl}_{3}\right)$

$153.20(\mathrm{C}(1)), 134.54(\mathrm{C}(3)), 107.55(\mathrm{C}(4)), 97.43(\mathrm{C}(2)), 57.83(\mathrm{C}(5)), 25.62$

(C(9)), $18.15(\mathrm{C}(6)), 10.65(\mathrm{C}(8)),-4.49(\mathrm{C}(7))$

IR: (neat)

3089 (w), 2958 (s), 2935 (s), 2896 (m), 2859 (s), 1658 (s), 1463 (m), 1440 (m), 1290 (s), 1255 (s), 1209 (s), 1170 (s), 1124 (s), 1043 (m)

MS: $\quad(\mathrm{CI}, 70 \mathrm{eV})$

$229\left(\mathrm{M}^{+}, 6\right), 213$ (4), 187 (100), 173 (12)

HRMS: calcd for $\mathrm{C}_{12} \mathrm{H}_{24} \mathrm{O}_{2} \mathrm{Si}$ : 229.1264, found: 229.1259

(Z)-tert-Butyl-(1-ethoxy-3-methyl-1,3-butadienyloxy)dimethylsilane ((Z)-3c)

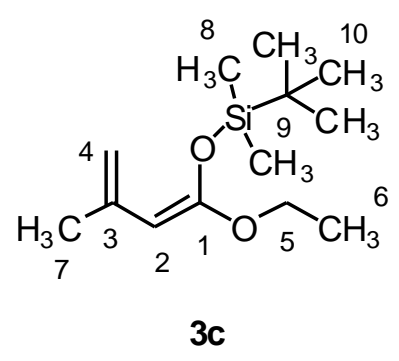

Following General Procedure 1, $1.54 \mathrm{~mL}$ of diisopropylamine (11 mmol, 1.1 equiv) was combined with $7.1 \mathrm{~mL}$ of $n$-BuLi (1.55 M in hexane, $11 \mathrm{mmol}, 1.1$ equiv), $2.1 \mathrm{~mL}$ of HMPA (12 mmol, 1.2 equiv), $1.39 \mathrm{~mL}$ of ethyl senecioate (10 mmol, 1.0 equiv.) and $1.65 \mathrm{~g}$ of TBSCl (11.6 mmol, 1.2 equiv) to yield, after short path distillation, $1.65 \mathrm{~g}(68 \%,>99 / 1 \mathrm{Z} / \mathrm{E})$ of $\mathbf{3 c}$ as a colorless oil. 
Data for $(Z)-3 \mathbf{c}:$

bp: $\quad 68{ }^{\circ} \mathrm{C}(1 \mathrm{~mm} \mathrm{Hg})$

매 NMR: $\quad\left(400 \mathrm{MHz}, \mathrm{CDCl}_{3}\right)$

$4.77(\mathrm{~d}, J=2.4,1 \mathrm{H}, \mathrm{HC}(4)), 4.51$ (d, $J=2.4,1 \mathrm{H}, \mathrm{HC}(4)), 4.2$ (s, $1 \mathrm{H}, \mathrm{HC}(2))$,

3.78 (q, $\left.J=6.8,2 \mathrm{H}, \mathrm{H}_{2} \mathrm{C}(5)\right), 1.95$ (s, $\left.3 \mathrm{H}, \mathrm{H}_{3} \mathrm{C}(7)\right), 1.29$ (t, $J=7.08,3 \mathrm{H}$, $\left.\mathrm{H}_{3} \mathrm{C}(6)\right), 0.94$ (s, $\left.9 \mathrm{H}, \mathrm{H}_{3} \mathrm{C}(10)\right), 0.20$ (s, $\left.6 \mathrm{H}, \mathrm{H}_{3} \mathrm{C}(8)\right)$

${ }^{13} \mathrm{C} \mathrm{NMR}: \quad\left(100 \mathrm{MHz}, \mathrm{CDCl}_{3}\right)$

156.62 (C(1)), $140.50(\mathrm{C}(3)), 107.02$ (C(4)), 80.39 (C(4)), 63.42 (C(2)), 25.85

$(\mathrm{C}(10)), 23.96(\mathrm{C}(6)), 18.14(\mathrm{C}(7)), 14.37(\mathrm{C}(9)),-3.70(\mathrm{C}(8))$

IR: (neat)

3079 (w), 2960 (m), 2931 (s), 2859 (m), 1650 (s), 1606 (w), 1473 (m), 1452 (m), 1349 (m), 1375 (m), 1275 (m), 1195 (s), 1087 (s), 1031 (m)

MS: $\quad(\mathrm{CI}, 70 \mathrm{eV})$

$243\left(\mathrm{M}^{+}, 41\right), 201$ (73), 185 (46), 147 (38), 127 (29), 103 (70), 75 (100)

HRMS: calcd for $\mathrm{C}_{13} \mathrm{H}_{26} \mathrm{O}_{2} \mathrm{Si}$ : 243.1780, found: 243.1403

(1Z,3Z)-[1-(tert-Butyloxy)-1,3-pentadienyloxy]-tert-butyldimethylsilane ((1Z,3Z)-3d)

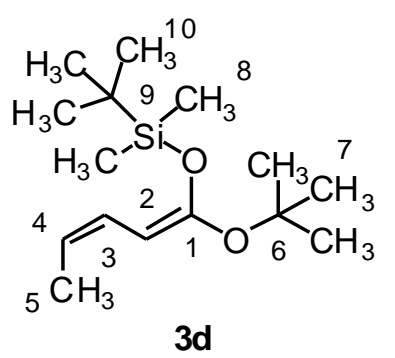

Following General Procedure 1, $1.54 \mathrm{~mL}$ of diisopropylamine (11 mmol, 1.1 equiv) was combined with $7.1 \mathrm{~mL}$ of $n-\mathrm{BuLi}$ (1.55 $\mathrm{M}$ in hexane, $11 \mathrm{mmol}, 1.1$ equiv), $2.1 \mathrm{~mL}$ of HMPA (12 mmol, 1.2 equiv), $1.55 \mathrm{~g}$ of tert-butyl 2-pentenoate (10 mmol, 1.0 equiv.) and $1.65 \mathrm{~g}$ of TBSCl (11.6 mmol, 1.2 equiv) to yield, after short path distillation, $1.41 \mathrm{~g}(52 \%, 89 \% 1 Z, 3 Z)$ of $\mathbf{3 d}$ as a colorless oil. 
Data for (1Z,3Z)-3d:

bp: $\quad 68{ }^{\circ} \mathrm{C}(1 \mathrm{~mm} \mathrm{Hg})$

${ }^{1} \mathrm{H}$ NMR: $\quad\left(500 \mathrm{MHz}, \mathrm{CDCl}_{3}\right)$

$6.12(\mathrm{dt}, J=1.7,10.7,1 \mathrm{H}, \mathrm{HC}(3)), 5.14(\mathrm{dq}, J=10.2,6.3,1 \mathrm{H}, \mathrm{HC}(4)), 4.87$ (d, $J$ $=10.8,1 \mathrm{H}, \mathrm{HC}(2)), 1.65\left(\mathrm{dd}, J=6.8,1.5,3 \mathrm{H}, \mathrm{H}_{3} \mathrm{C}(5)\right), 1.37\left(\mathrm{~s}, 9 \mathrm{H}, \mathrm{H}_{3} \mathrm{C}(7)\right)$, 0.95 (s, $\left.9 \mathrm{H}, \mathrm{H}_{3} \mathrm{C}(10)\right), 0.17$ (s, $\left.6 \mathrm{H}, \mathrm{H}_{3} \mathrm{C}(8)\right)$

NOE ${ }^{1} \mathrm{H}$ NMR: $\left(500 \mathrm{MHz}, \mathrm{CDCl}_{3}\right)$

Irradiation at $4.87 \mathrm{ppm}(\mathrm{HC}(2))$ enhanced signal at $1.65 \mathrm{ppm}(\mathrm{HC}(5))$ in major isomer

${ }^{13} \mathrm{C} \mathrm{NMR}: \quad\left(100 \mathrm{MHz}, \mathrm{CDCl}_{3}\right)$

153.22 (C(1)), 124.73 (C(3)), 118.08 (C(4)), 88.38 (C(6)), 79.39 (C(2)), 29.09

(C(5)), $28.50(\mathrm{C}(7)), 18.06(\mathrm{C}(10)), 13.25(\mathrm{C}(9)),-4.13(\mathrm{C}(8))$

IR: (neat)

2977 (s), 2958 (s), 2931 (s), 2859 (s), 1648 (s), 1610 (s), 1473 (m), 1367 (m), 1319 (m), 1299 (m), 1253 (s), 1201 (m), 1132 (s), 1062 (m)

MS: $\quad(\mathrm{EI}, 70 \mathrm{eV})$

270 (M, 6), 231 (5), 214 (100), 198 (42), 156 (38), 82 (98)

HRMS: calcd for $\mathrm{C}_{15} \mathrm{H}_{30} \mathrm{O}_{2} \mathrm{Si}: 270.2015$, found: 270.2020

tert-Butyl-(2,2-dimethyl-6-methylene-6H-[1,3]dioxin-4-yloxy)dimethylsilane (6)

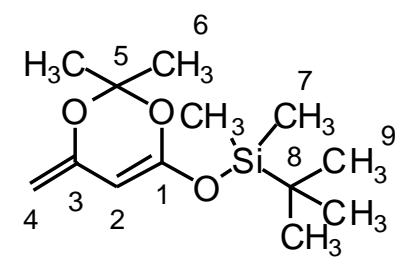

6

Following General Procedure 1, $1.54 \mathrm{~mL}$ of diisopropylamine (11 mmol, 1.1 equiv) was combined with $7.1 \mathrm{~mL}$ of $n-\mathrm{BuLi}$ (1.55 $\mathrm{M}$ in hexane, $11 \mathrm{mmol}, 1.1$ equiv), $2.1 \mathrm{~mL}$ of HMPA (12 mmol, 1.2 equiv), $1.3 \mathrm{~mL}$ of dioxanone (10 mmol, 1.0 equiv.) and $1.65 \mathrm{~g}$ of TBSCl (11.6 mmol, 1.2 equiv) to yield, after short path distillation, $1.52 \mathrm{~g}(59 \%)$ of $\mathbf{6}$ as a colorless oil. 
Data for 6:

bp: $\quad 78{ }^{\circ} \mathrm{C}(1 \mathrm{~mm} \mathrm{Hg})$

${ }^{1} \mathrm{H}$ NMR: $\quad\left(400 \mathrm{MHz}, \mathrm{CDCl}_{3}\right)$

4.65 (s, $1 \mathrm{H}, \mathrm{HC}(2)), 4.05$ (s, $1 \mathrm{H}, \mathrm{HC}(4)), 3.86$ (s, $1 \mathrm{H}, \mathrm{HC}(4)), 1.54$ (s, $6 \mathrm{H}$, $\mathrm{HC}(6)), 0.93$ (s, $\left.9 \mathrm{H}, \mathrm{H}_{3} \mathrm{C}(9)\right), 0.20$ (s, $6 \mathrm{H}, \mathrm{H}_{3} \mathrm{C}(7)$ )

${ }^{13} \mathrm{C} \mathrm{NMR}: \quad\left(100 \mathrm{MHz}, \mathrm{CDCl}_{3}\right)$

153.54 (C(1)), $151.94(\mathrm{C}(3)), 102.45(\mathrm{C}(5)), 84.67$ (C(4)), $76.64(\mathrm{C}(2)), 25.49$ $(\mathrm{C}(9)), 24.50(\mathrm{C}(6)), 18.01(\mathrm{C}(8)),-4.28(\mathrm{C}(7))$

IR: (neat)

2996 (m), 2958 (m), 2933 (m), 2888 (w), 2861 (m), 1668 (s), 1633 (m), 1473 (w), 1386 (m), 1375 (m), 1344 (s), 1294 (m), 1261 (s), 1224 (s), 1205 (s), 1126 (m), $1031(\mathrm{~m})$

MS: $\quad(\mathrm{CI}, 70 \mathrm{eV})$ $257\left(\mathrm{M}^{+}, 100\right), 215$ (21), 173 (22), 143 (24), 85 (29)

HRMS: calcd for $\mathrm{C}_{13} \mathrm{H}_{24} \mathrm{O}_{3} \mathrm{Si}$ : 257.1573, found: 257.1575

\section{General Procedure 2. Addition of Silyl Dienol Ethers to Conjugated Aldehydes.}

Preparation of $(2 E, 5 R)$ 5-Hydroxy-5-phenyl-2-pentenoic Acid Ethyl Ester ((2E,5R)-4aa)

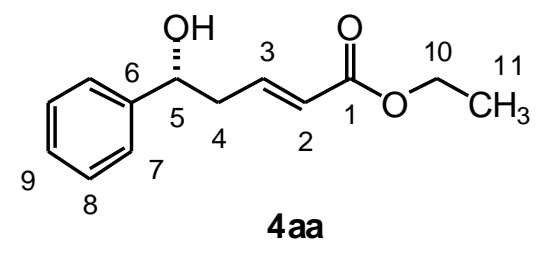

A flame-dried, $25 \mathrm{~mL}, 2$-neck flask containing a solution of $8.4 \mathrm{mg}$ of bis-phosphoramide $(R, R)-5(0.01 \mathrm{mmol}, 0.01$ equiv) and $102 \mu \mathrm{L}$ of benzaldehyde 1a (1.0 mmol, 1.0 equiv) in $\mathrm{CH}_{2} \mathrm{Cl}_{2}(5 \mathrm{~mL})$ was cooled to $-78{ }^{\circ} \mathrm{C}$ under nitrogen. To the resulting solution was added $123 \mu \mathrm{L}$ of $\mathrm{SiCl}_{4}$ (1.1 mmol, 1.1 equiv). Then $272 \mathrm{mg}$ of $\mathbf{3 a}$ (1.2 mmol, 1.2 equiv) was added dropwise over $5 \mathrm{~min}$ to the reaction mixture. The solution was allowed to stir at $-78{ }^{\circ} \mathrm{C}$ (bath temperature) for $3 \mathrm{~h}$ whereupon the cold reaction mixture was poured into a rapidly stirring 1/1 sat. aq. KF/1.0 
$\mathrm{M} \mathrm{KH} \mathrm{KHO}_{4}$ solution $(20 \mathrm{~mL})$. This biphasic mixture was stirred vigorously for $1 \mathrm{~h}$ before filtration through Celite. The aqueous layer was washed with $\mathrm{CH}_{2} \mathrm{Cl}_{2}(3 \times 25 \mathrm{~mL})$. The combined organic extracts were washed with brine $(50 \mathrm{~mL})$, dried over $\mathrm{Na}_{2} \mathrm{SO}_{4}(2 \mathrm{~g})$, filtered and the filtrate was concentrated in vacuo. The residue was purified by column chromatography (silica gel, hexanes/EtOAc, 4/1) to yield $196 \mathrm{mg}(89 \%)$ of $(2 E, 5 R)-4 \mathbf{a a}^{4}$ as a colorless oil.

\section{Data for $(2 E, 5 R)-4 a a:$}

${ }^{1} \mathrm{H}$ NMR: $\quad\left(400 \mathrm{MHz}, \mathrm{CDCl}_{3}\right)$

7.4-7.25 (m, $5 \mathrm{H}, \mathrm{HC}(7,8,9)), 6.95(\mathrm{dt}, J=15.6,7.1,1 \mathrm{H}, \mathrm{HC}(3)), 5.87(\mathrm{~d}, J=$ 15.6, $1 \mathrm{H}, \mathrm{HC}(2)), 4.81$ (m, $1 \mathrm{H}, \mathrm{HC}(5)), 4.16$ (q, J = 7.1, $\left.2 \mathrm{H}, \mathrm{H}_{2} \mathrm{C}(10)\right), 2.64$ (m, $\left.2 \mathrm{H}, \mathrm{H}_{2} \mathrm{C}(4)\right), 2.2$ (bs, $\left.1 \mathrm{H}, \mathrm{OH}\right), 1.26$ (t, $\left.J=7.1,3 \mathrm{H}, \mathrm{H}_{3} \mathrm{C}(11)\right)$

${ }^{13} \mathrm{C} \mathrm{NMR}: \quad\left(100 \mathrm{MHz}, \mathrm{CDCl}_{3}\right)$

166.29 (C(1)), 144.67 (C(3)), 142.97 (C(6)), 128.60 (C(8)), 127.89 (C(9)), 125.69 (C(7)), 124.0 (C(2)), 73.06 (C(5)), 60.29 (C(10)), 41.83 (C(4)), 14.21 (C(11))

IR: (neat)

3440 (m), 3029 (w), 2983 (m), 2939 (w), 2904 (w), 1712 (s), 1654 (s), 1494 (m), 1454 (m), 1369 (m), 1315 (s), 1278 (s), 1199 (s), 1160 (s), 1043 (s)

MS: $\quad(\mathrm{EI}, 70 \mathrm{eV})$

$221\left(\mathrm{M}^{+}, 3\right), 203$ (32), 175 (3), 129 (11), 114 (100), 107 (87), 86 (37)

Opt. Rot.: $\quad[\alpha]_{\mathrm{D}}^{24}+22.86(\mathrm{c}=1.0, \mathrm{EtOH})$, lit. $[\alpha]_{\mathrm{D}}^{24}+10.4(\mathrm{c}=1.13, \mathrm{EtOH})$ for $(5 R)-4 \mathbf{a a}^{4}$

TLC: $\quad R_{f} 0.26$ (hexanes/EtOAc, 4/1) [UV(254)/ $\left.\mathrm{KMnO}_{4}\right]$

SFC: $\quad t_{R}(R)$-4aa, $3.25(98.8 \%) ; t_{R}(S)$-4aa, 4.17 (1.2\%) (Chiralpak AD, 125 psi, $40{ }^{\circ} \mathrm{C}$, $10.0 \% \mathrm{CH}_{3} \mathrm{OH}$ in $\mathrm{CO}_{2}, 3.0 \mathrm{~mL} / \mathrm{min}, 220 \mathrm{~nm}$ )

Analysis: $\quad \mathrm{C}_{13} \mathrm{H}_{16} \mathrm{O}_{3}(220.26)$
Calcd:
C, 70.89;
H, $7.32 \%$
Found:
C, 70.64;
H, $7.28 \%$ 


\section{(2E,5R,6E)-5-Hydroxy-7-phenyl-2,6-heptadienoic Acid Ethyl Ester ((2E,5R,6E)-4ab)}

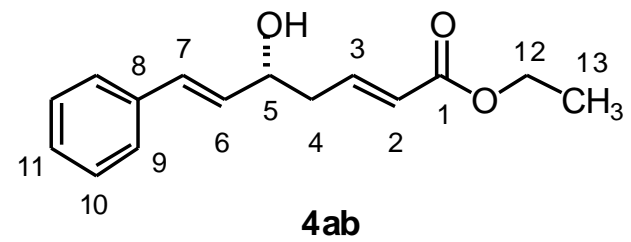

Following General Procedure 2, $8.4 \mathrm{mg}$ of bis-phosphoramide $(R, R)-5(0.01 \mathrm{mmol}, 0.01$ equiv) was combined with $126 \mu \mathrm{L}$ of cinnamaldehyde $\mathbf{1 b}$ (1.0 mmol, 1.0 equiv), $123 \mu \mathrm{L}$ of $\mathrm{SiCl}_{4}$ (1.1 mmol, 1.1 equiv) and $272 \mathrm{mg}$ of $\mathbf{3 a}$ (1.2 mmol, 1.2 equiv) to yield, after column chromatography (silica gel, hexanes/EtOAc, 4/1), $207 \mathrm{mg}(84 \%)$ of $(2 E, 5 R, 6 E)-\mathbf{4 a b}$ as a colorless oil.

Data for $(2 E, 5 R, 6 E)-\mathbf{4 a b}$ :

1․ NMR: $\quad\left(400 \mathrm{MHz}, \mathrm{CDCl}_{3}\right)$

7.4-7.2 (m, $5 \mathrm{H}, \mathrm{HC}(9,10,11)), 7.0$ (dt, $J=15.6,7.3,1 \mathrm{H}, \mathrm{HC}(3)), 6.62$ (d, $J=$ 16.1, $1 \mathrm{H}, \mathrm{HC}(7)), 6.23(\mathrm{dd}, J=15.9,6.6,1 \mathrm{H}, \mathrm{HC}(6)), 5.94(\mathrm{dt}, J=15.6,1.5,1$ $\mathrm{H}, \mathrm{HC}(2)), 4.45(\mathrm{q}, J=6.1,1 \mathrm{H}, \mathrm{HC}(5)), 4.18\left(\mathrm{q}, J=7.1,2 \mathrm{H}, \mathrm{H}_{2} \mathrm{C}(12)\right), 2.55$ (t, $J$ $\left.=7.1,2 \mathrm{H}, \mathrm{H}_{2} \mathrm{C}(4)\right), 1.28\left(\mathrm{t}, J=7.08,3 \mathrm{H}, \mathrm{H}_{3} \mathrm{C}(13)\right)$

${ }^{13} \mathrm{C} \mathrm{NMR:} \quad\left(100 \mathrm{MHz}, \mathrm{CDCl}_{3}\right)$

166.24 (C(1)), 144.17 (C(3)), 136.24 (C(8)), 131.13 (C(7)), 130.87 (C(6)), 128.61 (C(9)), 127.92 (C(11)), 126.54 (C(10)), 124.24 (C(2)), 71.53 (C(5)), 60.33 (C(12)), 40.12 (C(4)), 14.23 (C(13))

IR: (neat)

3430 (m), 3027 (w), 2983 (m), 2937 (w), 2904 (w), 1716 (s), 1654 (m), 1494 (w), 1448 (w), 1369 (m), 1317 (m), 1272 (m), 1201 (m), 1164 (m), 1041 (m)

MS: $\quad(E I, 70 \mathrm{eV})$

$245\left(\mathrm{M}^{-}, 3\right), 229$ (100), 183 (11), 155 (17), 133 (31), 115 (11)

Opt. Rot.: $\quad[\alpha]_{\mathrm{D}}^{24}-17.86(\mathrm{c}=1.0, \mathrm{EtOH})$

TLC: $\quad R_{f} 0.25$ (hexanes/EtOAc, 4/1) [UV(254)/KMnO $\mathrm{KM}_{4}$

SFC: $\quad t_{R}(R)-\mathbf{4 a b}, 4.15(98.3 \%) ; t_{R}(S)-4 a b, 4.41(1.7 \%)$ (Chiralpak AD, 125 psi, $40{ }^{\circ} \mathrm{C}$, $10.0 \% \mathrm{CH}_{3} \mathrm{OH}$ in $\mathrm{CO}_{2}, 3.0 \mathrm{~mL} / \mathrm{min}, 220 \mathrm{~nm}$ ) 
Analysis: $\quad \mathrm{C}_{15} \mathrm{H}_{18} \mathrm{O}_{3}(246.30)$
Calcd:
C, 73.15;
$\mathrm{H}, 7.37 \%$
Found:
C, 72.91;
$\mathrm{H}, 7.38 \%$

\section{General Procedure 3. Preparation of (2E,5S)-5-Hydroxy-7-phenyl-2-heptenoic Acid Ethyl Ester $((2 E, 5 S)-4 a c)$}

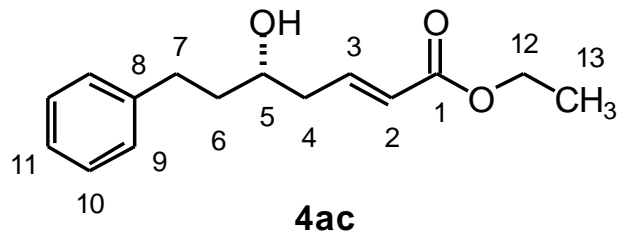

A flame-dried, $25 \mathrm{~mL}, 2$-neck flask containing a solution of $42 \mathrm{mg}$ of bis-phosphoramide $(R, R)-5$ (0.05 mmol, 0.05 equiv), $8 \mu \mathrm{L}$ of $i-\operatorname{Pr}_{2} \mathrm{EtN}(0.05 \mathrm{mmol}, 0.05$ equiv) and $132 \mu \mathrm{L}$ of hydrocinnamaldehyde $1 \mathrm{c}\left(1.0 \mathrm{mmol}, 1.0\right.$ equiv) in $\mathrm{CH}_{2} \mathrm{Cl}_{2}(5 \mathrm{~mL})$ was cooled to $-78{ }^{\circ} \mathrm{C}$ under nitrogen. To the resulting solution was added $123 \mu \mathrm{L}$ of $\mathrm{SiCl}_{4}(1.1 \mathrm{mmol}, 1.1$ equiv). Then 272 $\mathrm{mg}$ of 3a (1.2 mmol, 1.2 equiv) was added dropwise over $5 \mathrm{~min}$ to the reaction mixture. The solution was allowed to stir at $-78{ }^{\circ} \mathrm{C}$ (bath temperature) for $24 \mathrm{~h}$ whereupon the cold reaction mixture was poured into a rapidly stirring $1 / 1$ sat. aq. $\mathrm{KF} / 1.0 \mathrm{M} \mathrm{KH}_{2} \mathrm{PO}_{4}$ solution $(20 \mathrm{~mL})$.. This biphasic mixture was stirred vigorously for $1 \mathrm{~h}$ before filtration through Celite. The aqueous layer was washed with $\mathrm{CH}_{2} \mathrm{Cl}_{2}(3 \times 25 \mathrm{~mL})$. The combined organic extracts were washed with brine (50 mL), dried over $\mathrm{Na}_{2} \mathrm{SO}_{4}(2 \mathrm{~g})$, filtered and the filtrate was concentrated in vacuo. The residue was purified by column chromatography (silica gel, hexanes/EtOAc, 4/1) to yield 167 $\mathrm{mg}(68 \%)$ of $(2 E, 5 S)-4 \mathbf{a c}^{5}$ as a colorless oil.

\section{Data for $(2 E, 5 S)-\mathbf{4 a c}:$}

${ }^{1} \mathrm{H}$ NMR: $\quad\left(500 \mathrm{MHz}, \mathrm{CDCl}_{3}\right)$

7.3-7.18 (m, $5 \mathrm{H}, \mathrm{HC}(9,10,11)), 6.96(\mathrm{dt}, J=15.6,7.7,1 \mathrm{H}, \mathrm{HC}(3)), 5.9(\mathrm{dt}, J=$ 15.6, 1.5, $1 \mathrm{H}, \mathrm{HC}(2)), 4.19$ (q, $\left.J=7.3,2 \mathrm{H}, \mathrm{H}_{2} \mathrm{C}(12)\right), 3.78$ (m, $\left.1 \mathrm{H}, \mathrm{HC}(5)\right)$, 2.80-2.69 (m, 2 H, $\left.\mathrm{H}_{2} \mathrm{C}(4)\right)$ ) 2.42-2.35 (m, $\left.2 \mathrm{H}, \mathrm{H}_{2} \mathrm{C}(7)\right)$, 1.81 (m, $\left.2 \mathrm{H}, \mathrm{H}_{2} \mathrm{C}(6)\right)$, $1.28\left(\mathrm{t}, J=7.1,3 \mathrm{H}, \mathrm{H}_{3} \mathrm{C}(13)\right)$ 
${ }^{13} \mathrm{C}$ NMR: $\quad\left(125 \mathrm{MHz}, \mathrm{CDCl}_{3}\right)$

$166.27(\mathrm{C}(1)), 144.27(\mathrm{C}(3)), 141.58(\mathrm{C}(8)), 128.49(\mathrm{C}(9)), 128.41(\mathrm{C}(10))$, 125.99 (C(11)), 124.13 (C(2)), 69.79 (C(5)), 60.31 (C(12)), 40.31 (C(4)), 38.64 $(\mathrm{C}(7)), 31.92(\mathrm{C}(6)), 14.20(\mathrm{C}(13))$

IR: (neat)

3442 (m), 3025 (w), 2981 (w), 2935 (m), 2863 (w), 1718 (s), 1654 (m), 1496 (m), 1454 (m), 1369 (m), 1319 (m), 1270 (s), 1211 (m), 1176 (s), 1045 (s)

$\underline{\mathrm{MS}}$ : $\quad(\mathrm{EI}, 70 \mathrm{eV})$

$249\left(\mathrm{M}^{+}, 1\right), 230(15), 203(5), 156(7), 135(7), 114(80), 91(100)$

Opt. Rot.: $\quad[\alpha]_{\mathrm{D}}^{24}-12.77(\mathrm{c}=1.0, \mathrm{EtOH})$

TLC: $\quad R_{f} 0.21$ (hexanes/EtOAc, 4/1) [UV(254)/KMnO 4

SFC: $\quad t_{R}(R)$-4ac, $7.95(4.7 \%) ; t_{R}(S)$-4ac, 8.29 (95.3\%) (Chiralpak AD, 125 psi, $40{ }^{\circ} \mathrm{C}$, $7.0 \% \mathrm{CH}_{3} \mathrm{OH}$ in $\mathrm{CO}_{2}, 2.0 \mathrm{~mL} / \mathrm{min}, 220 \mathrm{~nm}$ )

Analysis: $\quad \mathrm{C}_{15} \mathrm{H}_{20} \mathrm{O}_{3}(248.32)$
Calcd:
C, 72.55;
H, $8.12 \%$
Found:
C, 72.44;
$\mathrm{H}, 8.23 \%$

\section{(2E,5R)-5-Hydroxy-2-methyl-5-phenyl-2-pentenoic Acid Methyl Ester ((2E,5R)-4ba)}

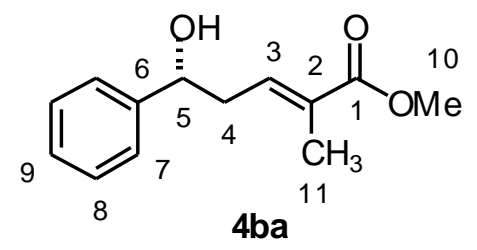

Following General Procedure 2, $8.4 \mathrm{mg}$ of bis-phosphoramide $(R, R)-5$ (0.01 mmol, 0.01 equiv) was combined with $102 \mu \mathrm{L}$ of benzaldehyde $1 \mathrm{a}\left(1.0 \mathrm{mmol}, 1.0\right.$ equiv), $123 \mu \mathrm{L}$ of $\mathrm{SiCl}_{4}$ (1.1 mmol, 1.1 equiv) and $272 \mathrm{mg}$ of $\mathbf{3 b}$ (1.2 mmol, 1.2 equiv) to yield, after column chromatography (silica gel, hexanes/EtOAc, 4/1), $205 \mathrm{mg}(93 \%)$ of (2E,5R)-4ba as a colorless oil. 


\section{Data for $(2 E, 5 R)-\mathbf{4 b a}$ :}

${ }^{1} \mathrm{H}$ NMR: $\quad\left(500 \mathrm{MHz}, \mathrm{CDCl}_{3}\right)$

7.4-7.28 (m, $5 \mathrm{H}, \mathrm{HC}(7,8,9)), 6.83(\mathrm{t}, J=7.5,1 \mathrm{H}, \mathrm{HC}(3)), 4.83(\mathrm{dd}, J=7.7,5.2$,

$1 \mathrm{H}, \mathrm{HC}(5)), 3.72$ (s, $\left.3 \mathrm{H}, \mathrm{H}_{3} \mathrm{C}(10)\right)$, 2.71-2.56 (m, $\left.2 \mathrm{H}, \mathrm{H}_{2} \mathrm{C}(4)\right), 2.0$ (s, $\left.1 \mathrm{H}, \mathrm{OH}\right)$, 1.79 (s, $3 \mathrm{H}, \mathrm{H}_{3} \mathrm{C}(11)$ )

${ }^{13} \mathrm{C} \mathrm{NMR}: \quad\left(125 \mathrm{MHz}, \mathrm{CDCl}_{3}\right)$

168.37 (C(1)), 143.17 (C(6)), 137.68 (C(3)), 129.95 (C(8)), 128.60 (C(9)), 127.88 $(\mathrm{C}(7)), 125.71(\mathrm{C}(2)), 73.46(\mathrm{C}(5)), 51.76(\mathrm{C}(10)), 38.43(\mathrm{C}(4)), 12.58(\mathrm{C}(11))$

IR: (neat)

3453 (m), 3029 (w), 2950 (m), 1710 (s), 1648 (m), 1436 (s), 1278 (s), 1195 (s), 1124 (s), 1083 (s), 1051 (s)

MS: $\quad(\mathrm{EI}, 70 \mathrm{eV})$

$221\left(\mathrm{M}^{+}, 15\right), 203$ (100), $171(20), 143$ (6), 114 (30)

Opt. Rot.: $\quad[\alpha]_{\mathrm{D}}^{24}+2.51(\mathrm{c}=1.0, \mathrm{EtOH})$

TLC: $\quad R_{f} 0.22$ (hexanes/EtOAc, 4/1) [UV(254)/KMnO $\left.\mathrm{KM}_{4}\right]$

SFC: $\quad t_{R}(S)$-4ba, $4.17(0.5 \%) ; t_{R}(R)-4 b a, 5.33(99.5 \%)$ (Chiralpak AD, 125 psi, $40{ }^{\circ} \mathrm{C}$, $7.0 \% \mathrm{CH}_{3} \mathrm{OH}$ in $\mathrm{CO}_{2}, 3.0 \mathrm{~mL} / \mathrm{min}, 220 \mathrm{~nm}$ )

Analysis: $\quad \mathrm{C}_{13} \mathrm{H}_{16} \mathrm{O}_{3}(220.20)$
Calcd:
C, 70.89;
H, $7.32 \%$
Found:
C, 70.74;
$\mathrm{H}, 7.36 \%$ 


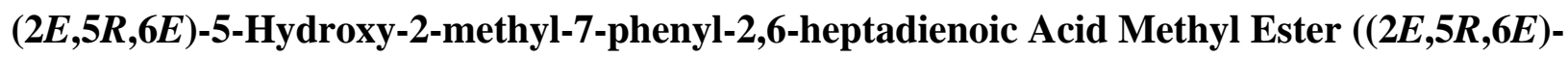
4bb)

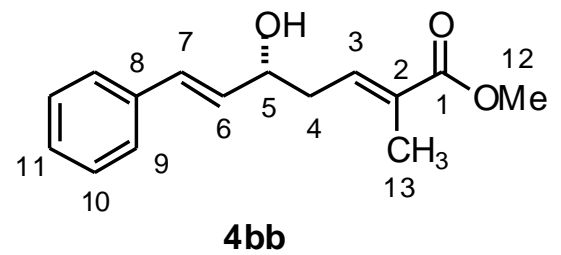

Following General Procedure 2, $8.4 \mathrm{mg}$ of bis-phosphoramide $(R, R)-5$ (0.01 mmol, 0.01 equiv) was combined with $126 \mu \mathrm{L}$ of cinnamaldehyde $\mathbf{1 b}\left(1.0 \mathrm{mmol}, 1.0\right.$ equiv), $123 \mu \mathrm{L}$ of $\mathrm{SiCl}_{4}$ (1.1 mmol, 1.1 equiv) and $272 \mathrm{mg}$ of $\mathbf{3 b}$ (1.2 mmol, 1.2 equiv) to yield, after column chromatography (silica gel, hexanes/EtOAc, 4/1), $216 \mathrm{mg}(88 \%)$ of $(2 E, 5 R, 6 E)-4 \mathbf{b b}$ as a colorless oil.

Data for $(2 E, 5 R, 6 E)-\mathbf{4 b b}$ :

1 $\mathrm{H}$ NMR: $\quad\left(500 \mathrm{MHz}, \mathrm{CDCl}_{3}\right)$

7.4-7.2 (m, $5 \mathrm{H}, \mathrm{HC}(9,10,11)), 6.85(\mathrm{t}, J=7.5,1 \mathrm{H}, \mathrm{HC}(3)), 6.63(\mathrm{~d}, J=15.9,1$ H, HC(7)), 6.24 (dd, $J=16.1,6.6,1 \mathrm{H}, \mathrm{HC}(6)), 4.45$ (q, $J=6.0,1 \mathrm{H}, \mathrm{HC}(5))$, 3.73 (s, $\left.3 \mathrm{H}, \mathrm{H}_{3} \mathrm{C}(12)\right), 2.53$ (m, $\left.2 \mathrm{H}, \mathrm{H}_{2} \mathrm{C}(4)\right)$ ), 1.88 (s, $\left.3 \mathrm{H}, \mathrm{H}_{3} \mathrm{C}(13)\right), 1.82$ (s, $1 \mathrm{H}$ $\mathrm{OH})$

${ }^{13} \mathrm{C} \mathrm{NMR}: \quad\left(125 \mathrm{MHz}, \mathrm{CDCl}_{3}\right)$

168.37 (C(1)), 137.28 (C(3)), 136.36 (C(8)), 131.16 (C(7)), 130.99 (C(6)), 130.04 $(\mathrm{C}(9)), 128.63(\mathrm{C}(11)), 127.88$ (C(10)), 126.55 (C(2)), 71.93 (C(5)), 51.29 (C(12)), 36.65 (C(13)), 12.75 (C(13))

IR: (neat) 3423 (m), 3025 (w), 2950 (m), 1710 (s), 1648 (m), 1494 (w), 1436 (m), 1282 (m), 1195 (m), 1128 (m), 1097 (m), 1081 (m), 1037 (m)

MS: $\quad(\mathrm{EI}, 70 \mathrm{eV})$

$245\left(\mathrm{M}^{-}, 4\right), 229$ (73), 197 (17), 169 (26), 133 (100), 115 (18)

Opt. Rot.: $\quad[\alpha]_{\mathrm{D}}^{24}-36.88(\mathrm{c}=1.0, \mathrm{EtOH})$

TLC: $\quad R_{f} 0.24$ (hexanes/EtOAc, 4/1) [UV(254)/KMnO $\mathrm{KM}_{4}$

SFC: $\quad t_{R}(S)-4 b b, 3.78(0.4 \%) ; t_{R}(R)-4 b b, 4.62$ (99.6\%) (Chiralpak AD, 125 psi, $40{ }^{\circ} \mathrm{C}$, $10.0 \% \mathrm{CH}_{3} \mathrm{OH}$ in $\mathrm{CO}_{2}, 3.0 \mathrm{~mL} / \mathrm{min}, 220 \mathrm{~nm}$ ) 
Analysis: $\quad \mathrm{C}_{15} \mathrm{H}_{18} \mathrm{O}_{3}(246.30)$

Calcd: $\quad$ C, $73.15 ; \quad H, 7.37 \%$

Found: $\quad$ C, $73.28 ; \quad$ H, $7.24 \%$

(2E,5R)-5-Hydroxy-3-methyl-5-phenyl-2-pentenoic Acid Ethyl Ester ((2E,5R)-4ca)

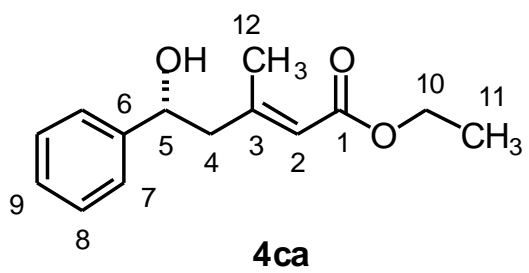

Following General Procedure 2, $8.4 \mathrm{mg}$ of bis-phosphoramide (R,R)-5 (0.01 mmol, 0.01 equiv) was combined with $102 \mu \mathrm{L}$ of benzaldehyde $1 \mathrm{a}\left(1.0 \mathrm{mmol}, 1.0\right.$ equiv), $123 \mu \mathrm{L}$ of $\mathrm{SiCl}_{4}$ (1.1 mmol, 1.1 equiv) and $290 \mathrm{mg}$ of $3 \mathrm{c}$ (1.2 mmol, 1.2 equiv) to yield, after column chromatography (silica gel, hexanes/EtOAc, 4/1), $214 \mathrm{mg}(91 \%)$ of $(2 E, 5 R)-4 \mathbf{c a}^{6}$ as a colorless oil.

Data for $(2 E, 5 R)-\mathbf{4 c a}$ :

${ }^{1} \mathrm{H}$ NMR: $\quad\left(500 \mathrm{MHz}, \mathrm{CDCl}_{3}\right)$

7.37-7.26 (m, 5 H, HC(7,8,9)), 5.77 (s, 1 H, HC(2)), 4.90 (dd, J = 8.8, 4.6, 1 H, $\mathrm{HC}(5)), 4.15$ (q, $\left.J=7.0,2 \mathrm{H}, \mathrm{H}_{2} \mathrm{C}(10)\right), 2.57$ (dd, $\left.J=13.9,8.8,1 \mathrm{H}, \mathrm{HC}(4)\right), 2.51$ (dd, $J=13.7,4.4,1 \mathrm{H}, \mathrm{HC}(4)), 2.22$ (s, $\left.3 \mathrm{H}, \mathrm{H}_{3} \mathrm{C}(12)\right), 2.04$ (s, $\left.1 \mathrm{H}, \mathrm{OH}\right), 1.27$ (t, $\left.J=7.1,3 \mathrm{H}, \mathrm{H}_{3} \mathrm{C}(11)\right)$

NOE ${ }^{1} \mathrm{H}$ NMR: $\left(500 \mathrm{MHz}, \mathrm{CDCl}_{3}\right)$

Irradiation at $5.77 \mathrm{ppm}(\mathrm{HC}(2))$ enhanced signal at $2.57 \mathrm{ppm}(\mathrm{HC}(4))$ in major isomer

${ }^{13} \mathrm{C} \mathrm{NMR}: \quad\left(125 \mathrm{MHz}, \mathrm{CDCl}_{3}\right)$

166.44 (C(1)), 155.63 (C(3)), 143.69 (C(6)), 128.59 (C(8)), 127.85 (C(9)), 125.67 (C(7)), 118.55 (C(2)), 72.09 (C(5)), 59.64 (C(10)), 50.70 (C(4)), 18.94 (C(12)), $14.23(\mathrm{C}(11))$ 
IR: (neat)

3451 (m), 3029 (w), 2981 (m), 2940 (m), 2906 (m), 1710 (s), 1648 (s), 1452 (m), $1369(\mathrm{~m}), 1226(\mathrm{~s}), 1151$ (s), 1058 (s)

MS: $\quad(\mathrm{EI}, 70 \mathrm{eV})$

$235\left(\mathrm{M}^{+}, 7\right), 217$ (100), 189 (7), 171 (15), 128 (80), 107 (37), 100 (27), 83 (30)

Opt. Rot.: $\quad[\alpha]_{\mathrm{D}}^{24}+19.61(\mathrm{c}=1.0, \mathrm{EtOH})$

TLC: $\quad R_{f} 0.36$ (hexanes/EtOAc, 4/1) [UV(254)/KMnO $\left.\mathrm{KM}_{4}\right]$

SFC: $\quad t_{R}(R)$-4ca, $4.04(96.1 \%) ; t_{R}(S)$-4ca, 7.92 (3.9\%) (Chiralpak AD, 125 psi, $40{ }^{\circ} \mathrm{C}$, $7.5 \% \mathrm{CH}_{3} \mathrm{OH}$ in $\mathrm{CO}_{2}, 3.0 \mathrm{~mL} / \mathrm{min}, 220 \mathrm{~nm}$ )

Analysis: $\quad \mathrm{C}_{14} \mathrm{H}_{18} \mathrm{O}_{3}(234.29)$
Calcd:
C, 71.77;
$\mathrm{H}, 7.74 \%$
Found:
C, 71.65;
$\mathrm{H}, 7.98 \%$

(2E,5R,6E)-5-Hydroxy-3-methyl-7-phenyl-2,6-heptadienoic Acid Ethyl Ester ((2E,5R,6E)$4 \mathrm{cb})$

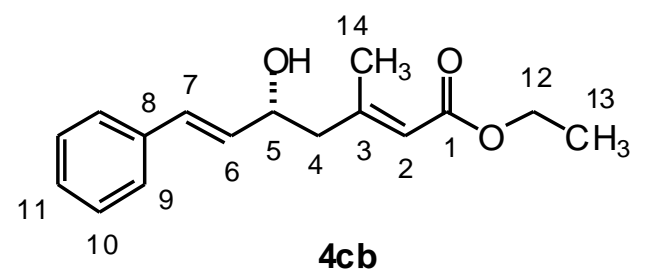

Following General Procedure 2, $8.4 \mathrm{mg}$ of bis-phosphoramide $(R, R)-5$ (0.01 mmol, 0.01 equiv) was combined with $126 \mu \mathrm{L}$ of cinnamaldehyde $\mathbf{1 b}\left(1.0 \mathrm{mmol}, 1.0\right.$ equiv), $123 \mu \mathrm{L}$ of $\mathrm{SiCl}_{4}$ (1.1 mmol, 1.1 equiv) and $290 \mathrm{mg}$ of $3 \mathbf{c}(1.2 \mathrm{mmol}, 1.2$ equiv) to yield, after column chromatography (silica gel, hexanes/EtOAc, 4/1), $253 \mathrm{mg}(97 \%, 97 / 3$ 2E/2Z) of $(2 E, 5 R, 6 E)-\mathbf{4 c b}$ as a colorless oil.

Data for $(2 E, 5 R, 6 E)-4 \mathbf{c b}$ :

${ }^{1} \mathrm{H}$ NMR: $\quad\left(500 \mathrm{MHz}, \mathrm{CDCl}_{3}\right)$

7.39-7.25 (m, $5 \mathrm{H}, \mathrm{HC}(9,10,11)), 6.63(\mathrm{~d}, J=15.8,1 \mathrm{H}, \mathrm{HC}(7)), 6.22(\mathrm{dd}, J=$ 16.0, 6.5, $1 \mathrm{H}, \mathrm{HC}(6)), 5.79$ (s, $1 \mathrm{H}, \mathrm{HC}(2)), 4.53$ (q, $J=6.7,1 \mathrm{H}, \mathrm{HC}(5)), 4.15$ (q, 
$\left.J=7.2,2 \mathrm{H}, \mathrm{H}_{2} \mathrm{C}(12)\right), 2.45\left(\mathrm{~d}, J=6.7,2 \mathrm{H}, \mathrm{H}_{2} \mathrm{C}(4)\right), 2.25$ (s, $\left.3 \mathrm{H}, \mathrm{H}_{3} \mathrm{C}(14)\right), 1.80$ (s, $1 \mathrm{H}, \mathrm{OH}), 1.28\left(\mathrm{t}, J=7.2,3 \mathrm{H}, \mathrm{H}_{3} \mathrm{C}(13)\right)$

NOE ${ }^{1} \mathrm{H}$ NMR: $\left(500 \mathrm{MHz}, \mathrm{CDCl}_{3}\right)$

Irradiation at $5.79 \mathrm{ppm}(\mathrm{HC}(2))$ enhanced signal at $2.45 \mathrm{ppm}(\mathrm{HC}(4))$ in major isomer

${ }^{13} \mathrm{C} \mathrm{NMR}: \quad\left(125 \mathrm{MHz}, \mathrm{CDCl}_{3}\right)$

$166.67(\mathrm{C}(1)), 155.57(\mathrm{C}(3)), 136.60(\mathrm{C}(8)) .131 .37(\mathrm{C}(7)), 130.99(\mathrm{C}(6)), 128.85$ (C(9)), 128.09 (C(11)), 126.77 (C(10)), 118.86 (C(2)), 70.77 (C(5)), 59.89 (C(12)), 49.01 (C(4)), 19.28 (C(14)), 14.47 (C(13))

IR: (neat)

3440 (m), 3025 (m), 2981 (m), 2939 (w), 2906 (w), 1712 (s), 1646 (m), 1494 (w), 1448 (m), 1382 (m), 1369 (m), 1295 (m), 1224 (s), 1149 (s), 1099 (m), 1058 (m)

MS: $\quad(\mathrm{EI}, 70 \mathrm{eV})$

260 (M, 1), 243 (2), 213 (2), 185 (2), 157 (5), 133 (100), 115 (18), 103 (21), 91 (13), 72 (24)

Opt. Rot.: $\quad[\alpha]_{\mathrm{D}}^{24}-9.94(\mathrm{c}=1.0, \mathrm{EtOH})$

TLC: $\quad R_{f} 0.32$ (hexanes/EtOAc, 4/1) [UV(254)/ $\left.\mathrm{KMnO}_{4}\right]$

SFC: $\quad t_{R}(R)-\mathbf{4 c b}, 4.10(94.0 \%) ; t_{R}(S)-4 \mathbf{c b}, 7.35$ (6.0\%) (Chiralpak AD, 125 psi, $40{ }^{\circ} \mathrm{C}$, $12.0 \% \mathrm{CH}_{3} \mathrm{OH}$ in $\mathrm{CO}_{2}, 3.0 \mathrm{~mL} / \mathrm{min}, 220 \mathrm{~nm}$ )

Analysis: $\quad \mathrm{C}_{16} \mathrm{H}_{20} \mathrm{O}_{3}(260.33)$
Calcd:
C, 73.82;
H, $7.74 \%$
Found:
C, 73.55;
$\mathrm{H}, 7.83 \%$ 


\section{(2E,5S)-5-Hydroxy-3-methyl-7-phenyl-2-Heptenoic Acid Ethyl Ester ((2E,5S)-4cc)}

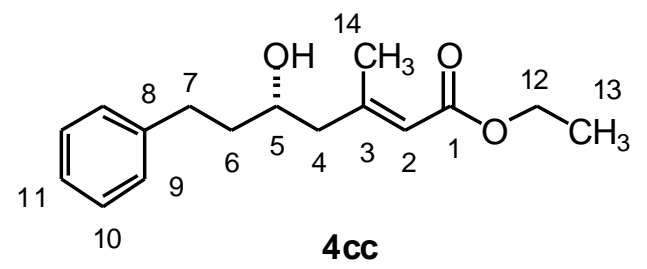

Following General Procedure 3, $42 \mathrm{mg}$ of bis-phosphoramide $(R, R)-5$ (0.05 mmol, 0.05 equiv) was combined with $8 \mu \mathrm{L}$ of $i$ - $\operatorname{Pr}_{2} \mathrm{EtN}(0.05 \mathrm{mmol}, 0.05$ equiv), $126 \mu \mathrm{L}$ of hydrocinnamaldehyde 1c (1.0 mmol, 1.0 equiv), $123 \mu \mathrm{L}$ of $\mathrm{SiCl}_{4}$ (1.1 mmol, 1.1 equiv) and 290 mg of 3c (1.2 mmol, 1.2 equiv) to yield, after column chromatography (silica gel, hexanes/EtOAc, 9/1), $190 \mathrm{mg}(73 \%)$ of (2E,5S)-4cc as a colorless oil.

Data for $(2 E, 5 S)-4 \mathrm{cc}$ :

${ }^{1} \mathrm{H}$ NMR: $\quad\left(500 \mathrm{MHz}, \mathrm{CDCl}_{3}\right)$

7.31-7.18 (m, $5 \mathrm{H}, \mathrm{HC}(9,10,11)), 5.74$ (s, $1 \mathrm{H}, \mathrm{HC}(2)), 4.15$ (q, $J=7.2,2 \mathrm{H}$ $\mathrm{H}_{2} \mathrm{C}(12)$ ), 3.84 (pent, $J=6.5,1 \mathrm{H}, \mathrm{HC}(5)$ ), 2.83 (dt, $J=13.7,7.9,1 \mathrm{H}, \mathrm{HC}(7)$ ), $2.70(\mathrm{dt}, J=13.8,8.3,1 \mathrm{H}, \mathrm{HC}(7)), 2.33(\mathrm{dd}, J=13.5,4.0,1 \mathrm{H}, \mathrm{HC}(4)), 2.27$ (dd, $J=13.5,8.6,1 \mathrm{H}, \mathrm{HC}(4)), 2.17$ (s, $\left.3 \mathrm{H}, \mathrm{H}_{3} \mathrm{C}(14)\right), 1.79$ (q, $\left.J=7.72,2 \mathrm{H}, \mathrm{H}_{2} \mathrm{C}(6)\right)$, 1.65 (s, $1 \mathrm{H}, \mathrm{OH}), 1.28$ (t, $\left.J=7.1,3 \mathrm{H}, \mathrm{H}_{3} \mathrm{C}(13)\right)$

NOE ${ }^{1} \mathrm{H}$ NMR: $\left(500 \mathrm{MHz}, \mathrm{CDCl}_{3}\right)$

Irradiation at $5.74 \mathrm{ppm}(\mathrm{HC}(2))$ enhanced signal at $2.27 \mathrm{ppm}(\mathrm{HC}(4))$ in major isomer

${ }^{13} \mathrm{C} \mathrm{NMR}: \quad\left(125 \mathrm{MHz}, \mathrm{CDCl}_{3}\right)$

$166.41(\mathrm{C}(1)), 156.06(\mathrm{C}(3)), 141.69$ (C(8)), 128.46 (C(9)), 128.40 (C(10)), 125.95 (C(11)), 118.32 (C(2)), 68.62 (C(5)), 59.65 (C(12)), 49.04 (C(4)), 38.86 (C(7)), 31.96 (C(6)), 18.92 (C(14)), 14.23 (C(13))

IR: (neat)

3450 (m), 3025 (w), 2981 (m), 2939 (m), 2863 (m), 1712 (s), 1644 (s), 1496 (m), 1454 (m), 1369 (m), 1280 (m), 1226 (s), 1149 (s), 1068 (m)

MS: $\quad(\mathrm{EI}, 70 \mathrm{eV})$

$263\left(\mathrm{M}^{+}, 1\right), 244$ (8), 217 (16), 171 (3), 128 (100), 117 (13), 100 (30), 91 (48), 82 
Opt. Rot.: $\quad[\alpha]_{\mathrm{D}}^{24}-5.85(\mathrm{c}=1.0, \mathrm{EtOH})$

TLC: $\quad R_{f} 0.51$ (hexanes/EtOAc, 4/1) [UV(254)/KMnO $\left.\mathrm{KM}_{4}\right]$

SFC: $\quad t_{R}(R)$-4cc, $3.52(97.5 \%) ; t_{R}(S)$-4cc, 4.18 (2.5\%) (Chiralpak AD, 125 psi, $40{ }^{\circ} \mathrm{C}$, $12.0 \% \mathrm{CH}_{3} \mathrm{OH}$ in $\mathrm{CO}_{2}, 3.0 \mathrm{~mL} / \mathrm{min}, 220 \mathrm{~nm}$ )

Analysis: $\quad \mathrm{C}_{16} \mathrm{H}_{22} \mathrm{O}_{3}(262.34)$
Calcd:
C, 73.25;
$\mathrm{H}, 8.45 \%$
Found:
C, 73.16;
$\mathrm{H}, 8.22 \%$

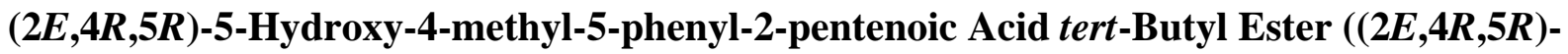
4da)

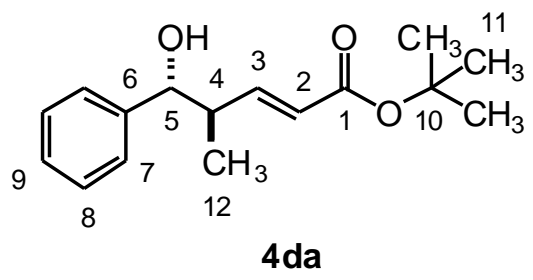

Following General Procedure 2, $8.4 \mathrm{mg}$ of bis-phosphoramide $(R, R)-5$ (0.01 mmol, 0.01 equiv) was combined with $102 \mu \mathrm{L}$ of benzaldehyde $1 \mathrm{a}$ (1.0 mmol, 1.0 equiv), $123 \mu \mathrm{L}$ of $\mathrm{SiCl}_{4}$ (1.1 mmol, 1.1 equiv) and $324 \mathrm{mg}$ of $\mathbf{3 d}$ (1.2 mmol, 1.2 equiv) to yield, after column chromatography (silica gel, hexanes/EtOAc, 4/1), $242 \mathrm{mg}(92 \%)$ of $(2 E, 4 R, 5 R)-4 \mathbf{d a}$ as a colorless oil.

Data for $(2 E, 4 R, 5 R)-\mathbf{4 d a}$ :

${ }^{1} \mathrm{H}$ NMR: $\quad\left(400 \mathrm{MHz}, \mathrm{CDCl}_{3}\right)$

7.37-7.29 (m, 5 H, HC(7,8,9)), 6.91 (dd, $J=15.6,8.3,1 \mathrm{H}, \mathrm{HC}(3)), 5.84$ (dd, $J=$ 15.9, 0.7, $1 \mathrm{H}, \mathrm{HC}(2)), 4.47$ (d, J= 7.6, $1 \mathrm{H}, \mathrm{HC}(5)), 2.62$ (m, $1 \mathrm{H}, \mathrm{HC}(4)), 2.65$ (s, $1 \mathrm{H}, \mathrm{OH}), 1.48$ (s, $\left.9 \mathrm{H}, \mathrm{H}_{3} \mathrm{C}(11)\right), 0.89$ (d, $\left.J=6.8,3 \mathrm{H}, \mathrm{H}_{3} \mathrm{C}(12)\right)$

${ }^{13} \mathrm{C}$ NMR: $\quad\left(100 \mathrm{MHz}, \mathrm{CDCl}_{3}\right)$ 165.74 (C(1)), 149.76 (C(3)), 142.17 (C(6)), 128.45 (C(8)), 127.95 (C(9)), 126.66 (C(7)), $124.11(\mathrm{C}(2)), 80.27$ (C(10)), 77.94 (C(5)), 44.28 (C(4)), 28.12 (C(11)), $16.17(\mathrm{C}(12))$ 
IR: (neat)

3442 (m), 2977 (s), 2933 (m), 1710 (s), 1650 (m), 1454 (m), 1367 (m), 1313

(m), $1253(\mathrm{~m}), 1157$ (s), $1035(\mathrm{~m})$

$\underline{\mathrm{MS}}: \quad(\mathrm{EI}, 70 \mathrm{eV})$

$263\left(\mathrm{M}^{+}, 29\right), 189$ (100), 143 (37), 100(39)

Opt. Rot.: $\quad[\alpha]_{\mathrm{D}}^{24}+65.37(\mathrm{c}=1.0, \mathrm{EtOH})$

TLC: $\quad R_{f} 0.45$ (hexanes/EtOAc, 4/1) [UV(254)/KMnO $\mathrm{KM}_{4}$

SFC: $\quad t_{R}(2 S, 3 R)-\mathbf{4 d a}, 6.20(94.5 \%) ; t_{R}(2 R, 3 S)-4 d a, 7.05(5.5 \%)$ (Chiralpak AD, 125 psi, $40{ }^{\circ} \mathrm{C}, 3.0 \% \mathrm{CH}_{3} \mathrm{OH}$ in $\mathrm{CO}_{2}, 3.0 \mathrm{~mL} / \mathrm{min}, 220 \mathrm{~nm}$ )

Analysis: $\quad \mathrm{C}_{16} \mathrm{H}_{22} \mathrm{O}_{3}(262.16)$

Calcd: $\quad$ C, $73.25 ; \quad H, 8.45 \%$

Found: $\quad$ C, $73.42 ; \quad H, 8.70 \%$

(2E,4R,5S)-5-Hydroxy-4-methyl-7-phenyl-2,6-Heptadienoic Acid tert-Butyl Ester $((2 E, 4 R, 5 S)-4 \mathrm{db})$

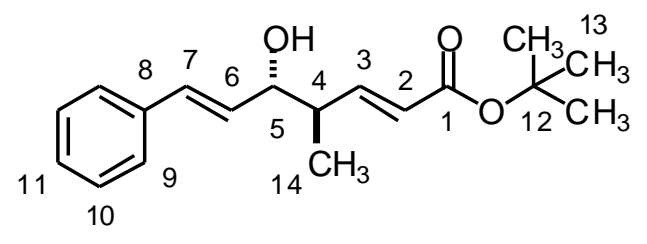

$4 d b$

Following General Procedure 2, $8.4 \mathrm{mg}$ of bis-phosphoramide $(R, R)-5$ (0.01 mmol, 0.01 equiv) was combined with $126 \mu \mathrm{L}$ of cinnamaldehyde $\mathbf{1 b}\left(1.0 \mathrm{mmol}, 1.0\right.$ equiv), $123 \mu \mathrm{L}_{\text {of } \mathrm{SiCl}_{4}}$ (1.1 mmol, 1.1 equiv) and $324 \mathrm{mg}$ of $\mathbf{3 d}$ (1.2 mmol, 1.2 equiv) to yield, after column chromatography (silica gel, hexanes/EtOAc, 9/1), $205 \mathrm{mg}(71 \%)$ of $(2 E, 4 R, 5 S)-\mathbf{4 d b}$ as a colorless oil.

Data for $(2 E, 4 R, 5 S)-\mathbf{4 d \mathbf { d b }}$ :

mp: $\quad 62-66{ }^{\circ} \mathrm{C}$ (hexanes, EtOAc)

${ }^{1} \mathrm{H}$ NMR: $\quad\left(400 \mathrm{MHz}, \mathrm{CDCl}_{3}\right)$

7.39-7.23 (m, $5 \mathrm{H}, \mathrm{HC}(9,10,11)), 6.90(\mathrm{dd}, J=16.1,8.0,1 \mathrm{H}, \mathrm{HC}(3)), 6.61$ (d, $J=$ 15.9, $1 \mathrm{H}, \mathrm{HC}(7)), 6.19$ (dd, $J=15.9,7.1,1 \mathrm{H}, \mathrm{HC}(6)), 5.85(\mathrm{dd}, J=15.6,1.0,1$ 
H, HC(2)), $4.16(\mathrm{t}, J=6.8,1 \mathrm{H}, \mathrm{HC}(5)), 2.52$ (m, $1 \mathrm{H}, \mathrm{HC}(4)), 1.78$ (s, $1 \mathrm{H}, \mathrm{OH})$, $1.48\left(\mathrm{~s}, 9 \mathrm{H}, \mathrm{H}_{3} \mathrm{C}(13)\right), 1.09$ (d, $\left.J=6.8,3 \mathrm{H}, \mathrm{H}_{3} \mathrm{C}(14)\right)$

${ }^{13} \mathrm{C} \mathrm{NMR}: \quad\left(100 \mathrm{MHz}, \mathrm{CDCl}_{3}\right)$

$165.81(\mathrm{C}(1)), 148.75(\mathrm{C}(3)), 136.28(\mathrm{C}(8)), 132.36(\mathrm{C}(7)), 129.65(\mathrm{C}(6)), 128.58$ (C(9)), 127.68 (C(11)), 126.59 (C(10)), 124.14 (C(2)), 80.33 (C(12)), 76.17 (C(5)), 42.91 (C(4)), 28.12 (C(13)), 15.66 (C(14))

IR: $\quad\left(\mathrm{CHCl}_{3}\right)$

3605 (w), 3027 (m), 3013 (m), 2981 (m), 1705 (s), 1651 (m), 1494 (m), 1455 (m), $1369(\mathrm{~m}), 1301(\mathrm{~m}), 1158(\mathrm{~s})$

MS: $\quad(\mathrm{CI}, 70 \mathrm{eV})$ $289\left(\mathrm{M}^{+}, 1\right), 271$ (33), 215 (100), 197 (9), 169 (10), 133 (21), 100 (3)

Opt. Rot.: $\quad[\alpha]_{\mathrm{D}}^{24}+5.78(\mathrm{c}=1.0, \mathrm{EtOH})$

TLC: $\quad R_{f} 0.58$ (hexanes/EtOAc, 4/1) [UV(254)/ $\left.\mathrm{KMnO}_{4}\right]$

SFC: $\quad t_{R}(2 R, 3 S)-\mathbf{4 d b}, 8.29(8.8 \%) ; t_{R}(2 S, 3 R)-4 \mathbf{d b}, 9.15(91.2 \%)$ (Chiralpak OJ, 125 psi, $40{ }^{\circ} \mathrm{C}, 4.0 \% \mathrm{CH}_{3} \mathrm{OH}$ in $\mathrm{CO}_{2}, 2.0 \mathrm{~mL} / \mathrm{min}, 220 \mathrm{~nm}$ )

Analysis: $\quad \mathrm{C}_{18} \mathrm{H}_{24} \mathrm{O}_{3}(288.38)$
Calcd:
C, 74.97;
$\mathrm{H}, 8.39 \%$
Found:
C, 75.11;
$\mathrm{H}, 8.59 \%$

(5R)-6-(2-Hydroxy-2-phenylethyl)-2,2-dimethyl-[1,3]-dioxin-4-one ((5R)-7a)

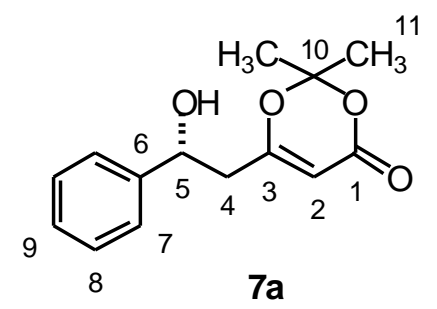

Following General Procedure 2, $8.4 \mathrm{mg}$ of bis-phosphoramide $(R, R)-5$ (0.01 mmol, 0.01 equiv) was combined with $102 \mu \mathrm{L}$ of benzaldehyde $1 \mathrm{a}\left(1.0 \mathrm{mmol}, 1.0\right.$ equiv), $123 \mu \mathrm{L}$ of $\mathrm{SiCl}_{4}$ (1.1 mmol, 1.1 equiv) and $307 \mathrm{mg}$ of $\mathbf{6}$ (1.2 mmol, 1.2 equiv) to yield, after column chromatography (silica gel, hexanes/EtOAc, 6/4), $228 \mathrm{mg}(92 \%)$ of $(5 R)-7 \mathbf{a}^{7}$ as a white, crystalline solid. 
Data for $(5 R)-7 \mathbf{a}$ :

mp: $\quad 70-72{ }^{\circ} \mathrm{C}$ (hexanes, EtOAc)

${ }^{1} \mathrm{H}$ NMR: $\quad\left(400 \mathrm{MHz}, \mathrm{CDCl}_{3}\right)$

7.4-7.28 (m, 5 H, HC(7,8,9)), 5.27 (s, 1 H, HC(2)), 4.98 (m, 1 H, HC(5)), 2.7 (dd, $J=14.6,8.2,1 \mathrm{H}, \mathrm{HC}(4)), 2.55(\mathrm{dd}, J=14.6,4.8,1 \mathrm{H}, \mathrm{HC}(4)), 2.1(\mathrm{~d}, J=4.0,1$ $\mathrm{H}, \mathrm{OH}), 1.65$ (s, $\left.3 \mathrm{H}, \mathrm{H}_{3} \mathrm{C}(11)\right), 1.63$ (s, $3 \mathrm{H}, \mathrm{H}_{3} \mathrm{C}(11)$ )

${ }^{13} \mathrm{C} \mathrm{NMR}: \quad\left(100 \mathrm{MHz}, \mathrm{CDCl}_{3}\right)$

168.19 (C(3)), 161.2 (C(1)), 142.65 (C(6)), 128.74 (C(8)), 128.33 (C(9)), 125.69 $(\mathrm{C}(7)), 106.66(\mathrm{C}(10)), 95.38(\mathrm{C}(2)), 71.22$ (C(5)), $43.16(\mathrm{C}(4)), 25.39$ (C(11)), $24.70(\mathrm{C}(11))$

MS: $\quad(\mathrm{CI}$, methane, $70 \mathrm{eV})$

$249\left(\mathrm{M}^{+}, 29\right), 191$ (28), 173 (32), 147 (22), 131 (100), 105 (25), 85 (21)

Opt. Rot.: $\quad[\alpha]_{\mathrm{D}}^{24}+35.1\left(\mathrm{c}=1.0, \mathrm{CHCl}_{3}\right)$, lit. $[\alpha]_{\mathrm{D}}^{24}+35.9\left(\mathrm{c}=1.0, \mathrm{CHCl}_{3}\right)$ for $(5 R)-7 \mathbf{a}^{7}$

TLC: $\quad R_{f} 0.35$ (hexanes/EtOAc, 6/4) [UV(254)/ $\left.\mathrm{KMnO}_{4}\right]$

SFC: $\quad t_{R}(S)-7 \mathbf{a}, 3.29(12.8 \%) ; t_{R}(R)-7 \mathbf{a}, 3.53(87.2 \%)$ (Chiralpak AD, 125 psi, $40{ }^{\circ} \mathrm{C}$, $10.0 \% \mathrm{CH}_{3} \mathrm{OH}$ in $\mathrm{CO}_{2}, 3.0 \mathrm{~mL} / \mathrm{min}, 220 \mathrm{~nm}$ )

\section{(5R)-6-(2-Hydroxy-4-phenyl 3-butenyl)-2,2-dimethyl-[1,3]-dioxin-4-one ((5R)-7b)}

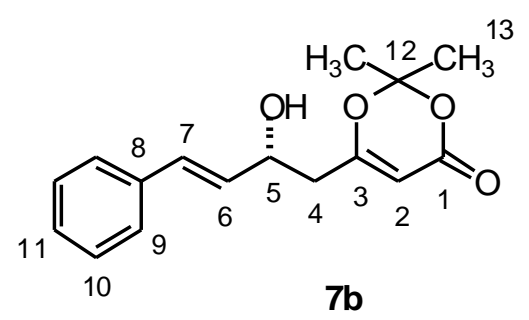

Following General Procedure 2, $8.4 \mathrm{mg}$ of bis-phosphoramide $(R, R)-5$ (0.01 mmol, 0.01 equiv) was combined with $126 \mu \mathrm{L}$ of cinnamaldehyde $\mathbf{1 b}\left(1.0 \mathrm{mmol}, 1.0\right.$ equiv), $123 \mu \mathrm{L}_{\text {of }} \mathrm{SiCl}_{4}$ (1.1 mmol, 1.1 equiv) and $290 \mathrm{mg}$ of $\mathbf{6}$ (1.2 mmol, 1.2 equiv) to yield, after column chromatography (silica gel, hexanes/EtOAc, 6/4), $241 \mathrm{mg}(88 \%)$ of $(5 R)-7 \mathbf{b}^{7}$ as a white, crystalline solid. 
Data for $(5 R)-7 \mathbf{b}$ :

mp: $\quad 81-82{ }^{\circ} \mathrm{C}$ (hexanes, EtOAc)

11 H NMR: $\quad\left(400 \mathrm{MHz}, \mathrm{CDCl}_{3}\right)$

7.37-7.25 (m, $5 \mathrm{H}, \mathrm{HC}(9,10,11)), 6.63(\mathrm{~d}, J=16.1,1 \mathrm{H}, \mathrm{HC}(7)), 6.21(\mathrm{dd}, J=$ 15.8, 6.9, $1 \mathrm{H}, \mathrm{HC}(6)), 5.36$ (s, $1 \mathrm{H}, \mathrm{HC}(2)), 4.6$ (q, J = 6.9, $1 \mathrm{H}, \mathrm{HC}(5)), 2.55$ (m, $\left.2 \mathrm{H}, \mathrm{H}_{2} \mathrm{C}(4)\right), 1.95$ (s, $\left.1 \mathrm{H}, \mathrm{OH}\right), 1.69$ (s, $\left.3 \mathrm{H}, \mathrm{H}_{3} \mathrm{C}(13)\right), 1.68$ (s, $3 \mathrm{H}, \mathrm{H}_{3} \mathrm{C}(13)$ )

${ }^{13} \mathrm{C}$ NMR: $\quad\left(100 \mathrm{MHz}, \mathrm{CDCl}_{3}\right)$

168.19 (C(3)), 161.03 (C(1)), 135.96 (C(8)), 131.62 (C(7)), 130.13 (C(6)), 128.72 (C(9)), 128.17 (C(11)), 126.55 (C(10)), 106.72 (C(12)), 95.43 (C(2)), 69.83 (C(5)), 41.50 (C(4)), 25.34 (C(13)), 24.84 (C(13))

MS: $\quad(\mathrm{EI}, 70 \mathrm{eV})$

$275\left(\mathrm{M}^{+}, 2\right), 216$ (45), 188 (60), 172 (23), 157 (18), 131 (44), 115 (32), 104 (100), $84(41)$

Opt. Rot.: $\quad[\alpha]_{\mathrm{D}}^{24}+14.43(\mathrm{c}=1.0, \mathrm{EtOH}),[\alpha]_{\mathrm{D}}^{24}+6.79\left(\mathrm{c}=1.0, \mathrm{CHCl}_{3}\right)$, lit $[\alpha]_{\mathrm{D}}^{24}+6.2(\mathrm{c}=1.0$, $\left.\mathrm{CHCl}_{3}\right)$ for $(5 R)-7 \mathbf{b}^{7}$

TLC: $\quad R_{f} 0.28$ (hexanes/EtOAc, 6/4) [UV(254)/ $\left.\mathrm{KMnO}_{4}\right]$

SFC: $\quad t_{R}(S)-7 \mathbf{b}, 6.56(11.1 \%) ; t_{R}(R)-7 \mathbf{b}, 7.9$ (88.9\%) (Chiralpak AD, 125 psi, $40{ }^{\circ} \mathrm{C}$, $8.0 \% \mathrm{CH}_{3} \mathrm{OH}$ in $\mathrm{CO}_{2}, 3.0 \mathrm{~mL} / \mathrm{min}, 220 \mathrm{~nm}$ )

(5S)-6-(2-Hydroxy-4-phenylbutyl)-2,2-dimethyl-[1,3]-dioxin-4-one ((5S)-7c)

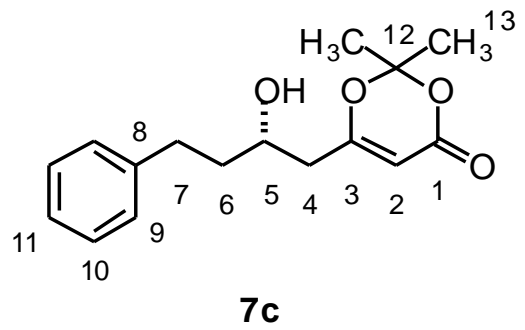

Following General Procedure 3, $42 \mathrm{mg}$ of bis-phosphoramide $(R, R)-5$ (0.05 mmol, 0.05 equiv) was combined with $8 \mu \mathrm{L}$ of $i$ - $\operatorname{Pr}_{2} \mathrm{EtN}(0.05 \mathrm{mmol}, 0.05$ equiv), $126 \mu \mathrm{L}$ of hydrocinnamaldehyde 1 c (1.0 mmol, 1.0 equiv), $123 \mu \mathrm{L}$ of $\mathrm{SiCl}_{4}(1.1 \mathrm{mmol}, 1.1$ equiv) and 272 
mg of 6 (1.2 mmol, 1.2 equiv) to yield, after column chromatography (silica gel, hexanes/EtOAc, 6/4), $230 \mathrm{mg}(83 \%)$ of $(5 S)-7 \mathbf{c}^{7}$ as a white, crystalline solid.

Data for $(5 S)-7 \mathbf{c}$ :

mp: $\quad 59-60{ }^{\circ} \mathrm{C}$ (hexanes, EtOAc)

${ }^{1} \mathrm{H}$ NMR: $\quad\left(500 \mathrm{MHz}, \mathrm{CDCl}_{3}\right)$

7.3-7.18 (m, $5 \mathrm{H}, \mathrm{HC}(9,10,11)$ ), 5.31 (s, $1 \mathrm{H}, \mathrm{HC}(2)), 3.92$ (pent, $J=6.2,1 \mathrm{H}$, HC(5)), 2.81 (m, $1 \mathrm{H}, \mathrm{HC}(4)), 2.70$ (m, $1 \mathrm{H}, \mathrm{HC}(4)), 2.42$ (dd, J = 14.4, 4.3, 1 H, $\mathrm{HC}(6)), 2.36$ (dd, $J=14.6,8.1,1 \mathrm{H}, \mathrm{HC}(6)), 1.82$ (m, 2 H, $\left.\mathrm{H}_{2} \mathrm{C}(7)\right), 1.68$ (s, $3 \mathrm{H}$, $\left.\mathrm{H}_{3} \mathrm{C}(12)\right), 1.66$ (s, $\left.3 \mathrm{H}, \mathrm{H}_{3} \mathrm{C}(12)\right)$

${ }^{13} \mathrm{C} \mathrm{NMR}: \quad\left(125 \mathrm{MHz}, \mathrm{CDCl}_{3}\right)$

168.95 (C(3)), 161.0 (C(1)), 141.27 (C(8)), 128.55 (C(9)), 128.37 (C(10)), 126.11 (C(11)), 106.63 (C(11)), 95.14 (C(2)), 68.32 (C(5)), 41.73 (C(4)), 38.89 (C(7)), $31.77(\mathrm{C}(6)), 25.16(\mathrm{C}(12)), 24.84(\mathrm{C}(12))$

MS: $\quad(\mathrm{EI}, 70 \mathrm{eV})$

$277\left(\mathrm{M}^{+}, 3\right), 218$ (28), 200 (31), 172 (7), 158 (16), 130 (24), 117 (44), 104 (22), $91(100), 69(26)$

Opt. Rot.: $\quad[\alpha]_{\mathrm{D}}^{24}+10.17(\mathrm{c}=1.0, \mathrm{EtOH}),[\alpha]_{\mathrm{D}}^{24}+3.4\left(\mathrm{c}=1.0, \mathrm{CHCl}_{3}\right)$, lit. $[\alpha]_{\mathrm{D}}^{24}+2.5(\mathrm{c}=1.0$, $\left.\mathrm{CHCl}_{3}\right)$ for $(5 S)-7 \mathbf{c}^{7}$

TLC: $\quad R_{f} 0.27$ (hexanes/EtOAc, 6/4) [UV(254)/KMnO $\mathrm{KM}_{4}$

SFC: $\quad t_{R}(R)-7 \mathrm{c}, 4.80(5.4 \%) ; t_{R}(S)-7 \mathrm{c}, 5.18(94.6 \%)$ (Chiralpak AD, 125 psi, $40{ }^{\circ} \mathrm{C}$, $8.0 \% \mathrm{CH}_{3} \mathrm{OH}$ in $\mathrm{CO}_{2}, 3.0 \mathrm{~mL} / \mathrm{min}, 220 \mathrm{~nm}$ ) 
(3S)-3-Hydroxy-2,2-dimethyl-3-phenylpropanoic Acid Methyl Ester ((3S)-13)

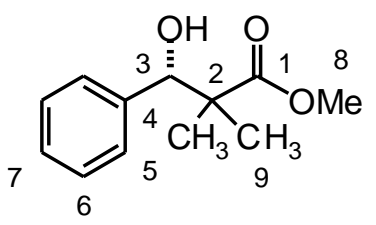

13

Following General Procedure 3, $42 \mathrm{mg}$ of bis-phosphoramide $(R, R)-5$ (0.05 mmol, 0.05 equiv) was combined with $8 \mu \mathrm{L}$ of $i-\operatorname{Pr}_{2} \operatorname{EtN}$ ( $0.05 \mathrm{mmol}, 0.05$ equiv), $102 \mu \mathrm{L}$ of benzaldehyde $1 \mathbf{a}\left(1.0 \mathrm{mmol}, 1.0\right.$ equiv), $123 \mu \mathrm{L}$ of $\mathrm{SiCl}_{4}(1.1 \mathrm{mmol}, 1.1$ equiv) and $208 \mathrm{mg}$ of $\mathbf{1 2}(1.2 \mathrm{mmol}$, 1.2 equiv) to yield, after Kugelrohr distillation, $180 \mathrm{mg}(87 \%)$ of $(3 S)-\mathbf{1 3}^{8}$ as a white, crystalline solid.

Data for $(3 S)-\mathbf{1 3}$ :

mp: $\quad 62-64{ }^{\circ} \mathrm{C}$

bp: $\quad 175^{\circ} \mathrm{C}(0.1 \mathrm{~mm} \mathrm{Hg}, \mathrm{ABT})$

${ }^{1} \mathrm{H}$ NMR: $\quad\left(400 \mathrm{MHz}, \mathrm{CDCl}_{3}\right)$

7.32-7.26 (m, $5 \mathrm{H}, \mathrm{HC}(5,6,7)), 4.90$ (s, $1 \mathrm{H}, \mathrm{HC}(3)), 3.72$ (s, $\left.3 \mathrm{H}, \mathrm{H}_{3} \mathrm{C}(8)\right), 3.05$

(s, $1 \mathrm{H}, \mathrm{OH}), 1.14$ (s, $\left.3 \mathrm{H}, \mathrm{H}_{3} \mathrm{C}(9)\right), 1.11$ (s, $\left.3 \mathrm{H}, \mathrm{H}_{3} \mathrm{C}(9)\right)$

${ }^{13} \mathrm{C} \mathrm{NMR}: \quad\left(100 \mathrm{MHz}, \mathrm{CDCl}_{3}\right)$

$178.21(\mathrm{C}(1)), 139.89(\mathrm{C}(4)), 127.77(\mathrm{C}(6)), 127.75(\mathrm{C}(5)), 127.63(\mathrm{C}(7)), 78.69$ (C(3)), $52.11(\mathrm{C}(8)), 47.67(\mathrm{C}(2)), 23.06(\mathrm{C}(9)), 19.0(\mathrm{C}(9))$

Opt. Rot.: $\quad[\alpha]_{\mathrm{D}}^{24}+1.94(\mathrm{c}=2.4, \mathrm{MeOH})$, lit. $[\alpha]_{\mathrm{D}}^{24}+5.7(\mathrm{c}=2.49, \mathrm{MeOH})$ for $(3 S)-\mathbf{1 3}^{8}$

TLC: $\quad R_{f} 0.36$ (hexanes/EtOAc, 4/1) [UV(254)/KMnO $\left.{ }_{4}\right]$

SFC: $\quad t_{R}(3 R)-13,3.69(91.1 \%) ; t_{R}(3 S)-13,4.25(8.9 \%)$ (Chiralpak OB, 125 psi, $40{ }^{\circ} \mathrm{C}$, $2.0 \% \mathrm{CH}_{3} \mathrm{OH}$ in $\mathrm{CO}_{2}, 2.0 \mathrm{~mL} / \mathrm{min}, 220 \mathrm{~nm}$ ) 


\section{Representative Procedure 4. Preparation of (3R)-1-Phenylpropane-1,3-diol ((3R)-14) from (2E,5R)-5-Hydroxy-5-phenyl-2-pentenoic Acid Ethyl Ester ((2E,5R)-4aa)}

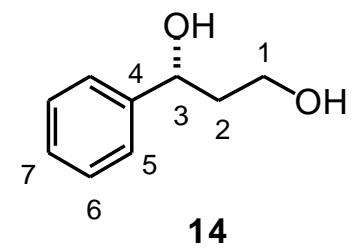

A $25 \mathrm{~mL}$, 1-neck flask containing a solution of $94 \mathrm{mg}(2 E, 5 R)-5$-hydroxy-5-phenyl-2pentenoic acid ethyl ester $(2 E, 5 R)-4 a a(0.42 \mathrm{mmol})$ in $5 \mathrm{~mL}$ of pentane was cooled to $-78{ }^{\circ} \mathrm{C}$ and a solution of ozone in oxygen was passed through it using a gas dispersion tube for $1 \mathrm{~h}$. The solution was sparged with $\mathrm{N}_{2}$ (to remove residual ozone) and then $2.89 \mathrm{~mL}$ of a solution of $\mathrm{LiAlH}_{4}$ in THF $\left(0.58 \mathrm{M}, 1.68 \mathrm{mmol}, 4\right.$ equiv) was added slowly at $-78{ }^{\circ} \mathrm{C}$. The flask was then fitted with a reflux condenser and was heated at $65{ }^{\circ} \mathrm{C}$ for $1 \mathrm{~h}$. The mixture was cooled to room temperature and $5 \mathrm{~mL}$ of sat. aq. sodium potassium tartrate solution was added and the mixture was stirred to break up the resulting precipitate. The organic layer was separated and the aqueous layer was washed with $\mathrm{Et}_{2} \mathrm{O}(3 \times 10 \mathrm{~mL})$. The combined organic extracts were washed with brine $(25 \mathrm{~mL})$, then were dried over $\mathrm{Na}_{2} \mathrm{SO}_{4}(5 \mathrm{~g})$, filtered and the filtrate was concentrated in vacuo. The residue was purified by column chromatography (silica gel, EtOAc/ hexanes, 2/1) to yield $41 \mathrm{mg}(64 \%)$ of $(3 R)-14^{9}$ as a colorless oil.

Data for $(3 R)-14:$

${ }^{1} \mathrm{H}$ NMR: $\quad\left(400 \mathrm{MHz}, \mathrm{CDCl}_{3}\right)$

7.36-7.25 (m, $5 \mathrm{H}, \mathrm{HC}(5,6,7)), 4.96(\mathrm{dd}, J=8.5,3.9,1 \mathrm{H}, \mathrm{HC}(3)), 3.86(\mathrm{t}, J=5.6$, $\left.2 \mathrm{H}, \mathrm{H}_{2} \mathrm{C}(1)\right), 2.55$ (s, $\left.1 \mathrm{H}, \mathrm{OH}\right), 2.1-1.85\left(\mathrm{~m}, 2 \mathrm{H}, \mathrm{H}_{2} \mathrm{C}(2)\right)$

Opt. Rot.: $\quad[\alpha]_{\mathrm{D}}^{24}+60.8\left(\mathrm{c}=1.0, \mathrm{CHCl}_{3}\right)$, lit. $[\alpha]_{\mathrm{D}}^{24}-63.0\left(\mathrm{c}=0.958, \mathrm{CHCl}_{3}\right)$ for $(3 S)-14^{9}$ TLC: $\quad R_{f} 0.28($ EtOAc/hexanes, 2/1) [p-anisaldehyde] 
(5R)-5-Hydroxy-7-phenylheptanoic Acid Ethyl Ester ((5R)-15) from (2E,5R,6E)-5-Hydroxy7-phenyl-2,6-heptadienoic Acid Ethyl Ester ((2E,5R,6E)-4ab)

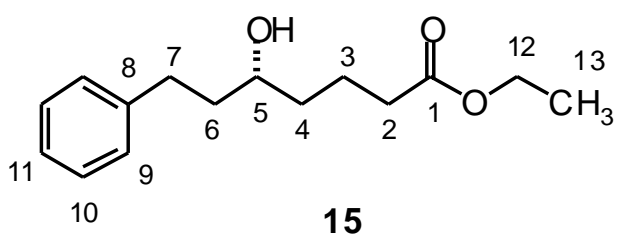

A $50 \mathrm{~mL}, 1$-neck flask containing a suspension of $246 \mathrm{mg}$ of $(2 E, 5 R, 6 E)-5$-hydroxy-7phenyl-2,6-heptadienoic acid ethyl ester $((2 E, 5 R, 6 E)-\mathbf{4 a b})(1.0 \mathrm{mmol})$ and $7.0 \mathrm{mg}$ of $10 \% \mathrm{Pd}-\mathrm{C}$ in $25 \mathrm{~mL}$ of EtOAc was stirred for $3 \mathrm{~h}$ at $23{ }^{\circ} \mathrm{C}$ under 1 atm of $\mathrm{H}_{2}$. After briefly evacuating the flask and venting with $\mathrm{N}_{2}$, the suspension was then filtered through Celite and the filtrate was concentrated in vасио. The residue was purified by column chromatography (silica gel, hexanes/EtOAc, 4/1) to yield $206 \mathrm{mg}(82 \%)$ of $(5 R)-\mathbf{1 5}$ as a colorless oil.

Data for $(5 R)-15:$

${ }^{1} \mathrm{H}$ NMR: $\quad\left(400 \mathrm{MHz}, \mathrm{CDCl}_{3}\right)$

7.30-7.16 (m, $5 \mathrm{H}, \mathrm{HC}(9,10,11)), 4.12$ (q, $\left.J=7.1,2 \mathrm{H}, \mathrm{H}_{2} \mathrm{C}(12)\right), 3.61$ (m, $1 \mathrm{H}$, $\mathrm{HC}(5)), 2.77$ (m, $1 \mathrm{H}, \mathrm{HC}(7)), 2.68$ (m, $1 \mathrm{H}, \mathrm{HC}(7)), 2.32(\mathrm{dt}, J=1.2,7.56,2 \mathrm{H}$, $\left.\mathrm{H}_{2} \mathrm{C}(2)\right)$, 1.79-1.67 (m, $4 \mathrm{H}, 2$ x $\left.\mathrm{H}_{2} \mathrm{C}(4,6)\right), 1.50$ (m, $2 \mathrm{H}, \mathrm{H}_{2} \mathrm{C}(3)$ ), 1.24 (t, J= 7.1, $\left.3 \mathrm{H}, \mathrm{H}_{3} \mathrm{C}(13)\right)$

${ }^{13} \mathrm{C} \mathrm{NMR}: \quad\left(100 \mathrm{MHz}, \mathrm{CDCl}_{3}\right)$

$173.78(\mathrm{C}(1)), 142.01(\mathrm{C}(8)), 128.37(\mathrm{C}(9)), 128.35(\mathrm{C}(10)), 125.29(\mathrm{C}(11))$, 70.65 (C(5)), 60.34 (C(12)), 39.63 (C(7)), 36.83 (C(4)), 34.05 (C(2)), 31.99 (C(6)), 20.84 (C(3)), 14.21 (C(13))

IR: (neat)

3451 (w), 2980 (w), 2866 (w), 1733 (s), 1495 (w), 1454 (m), 1373 (m), 1238 (m), $1180(\mathrm{~m}), 1094(\mathrm{~m}), 1031(\mathrm{~m})$

MS: $\quad(\mathrm{EI}, 70 \mathrm{eV})$

$251\left(\mathrm{M}^{+}, 1\right), 232$ (35), 204 (7), 186 (70), 117 (35), 104 (40), 91 (100), 71 (19)

Opt. Rot.: $\quad[\alpha]_{\mathrm{D}}^{24}-5.94(\mathrm{c}=1.0, \mathrm{EtOH})$

TLC: $\quad R_{f} 0.19$ (hexanes/EtOAc, 4/1) [UV(254)/ $\left.\mathrm{KMnO}_{4}\right]$ 
Analysis: $\quad \mathrm{C}_{15} \mathrm{H}_{22} \mathrm{O}_{3}(250.33)$
Calcd:
C, 71.97;
$\mathrm{H}, 8.86 \%$
Found:
C, 71.91;
$\mathrm{H}, 8.91 \%$

(5R)-5-Hydroxy-7-phenylheptanoic Acid Ethyl Ester (5R)-15 from (2E,5S)-5-Hydroxy-7phenyl-2-heptenoic Acid Ethyl Ester (2E,5S)-4ac

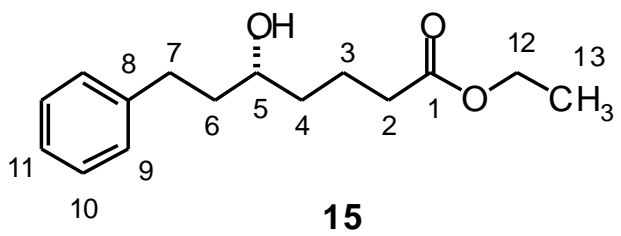

A $50 \mathrm{~mL}, 1$-neck flask containing a suspension of $50 \mathrm{mg}$ of $(2 E, 5 S)-5$-Hydroxy-7-phenyl2-heptenoic acid ethyl ester $(2 E, 5 S)-4 a c(0.2 \mathrm{mmol})$ and $1.4 \mathrm{mg}$ of $10 \% \mathrm{Pd}-\mathrm{C}$ in $5 \mathrm{~mL}$ of EtOAc was stirred for $3 \mathrm{~h}$ at $23{ }^{\circ} \mathrm{C}$ under 1 atm of $\mathrm{H}_{2}$. After briefly evacuating the flask and venting with $\mathrm{N}_{2}$, the suspension was then filtered through Celite and the filtrate was concentrated in vacuo. The residue was purified by column chromatography (silica gel, hexanes/EtOAc, 4/1) to yield 31 $\mathrm{mg}(61 \%)$ of $(5 R)-\mathbf{1 5}$ as a colorless oil.

\section{Data for $(5 R)-15$ :}

${ }^{1} \mathrm{H}$ NMR: $\quad\left(400 \mathrm{MHz}, \mathrm{CDCl}_{3}\right)$

7.30-7.16 (m, $5 \mathrm{H}, \mathrm{HC}(9,10,11)), 4.12$ (q, $\left.J=7.1,2 \mathrm{H}, \mathrm{H}_{2} \mathrm{C}(12)\right), 3.61$ (m, $1 \mathrm{H}$, $\mathrm{HC}(5)), 2.77$ (m, $1 \mathrm{H}, \mathrm{HC}(7)), 2.68$ (m, $1 \mathrm{H}, \mathrm{HC}(7)), 2.32$ (dt, $J=1.2,7.56,2 \mathrm{H}$, $\left.\mathrm{H}_{2} \mathrm{C}(2)\right)$, 1.79-1.67 (m, $4 \mathrm{H}, 2$ x $\left.\mathrm{H}_{2} \mathrm{C}(4,6)\right), 1.50$ (m, $2 \mathrm{H}, \mathrm{H}_{2} \mathrm{C}(3)$ ), 1.24 (t, J= 7.1, $\left.3 \mathrm{H}, \mathrm{H}_{3} \mathrm{C}(13)\right)$

Opt. Rot.: $\quad[\alpha]_{\mathrm{D}}^{24}-6.09(\mathrm{c}=1.0, \mathrm{EtOH})$ 
(5S)-5-Phenylpentane-1,3-diol ((5S)-16) from (2E,5S)-5-Hydroxy-7-phenyl-2-heptenoic Acid Ethyl Ester ((2E,5S)-4ac)

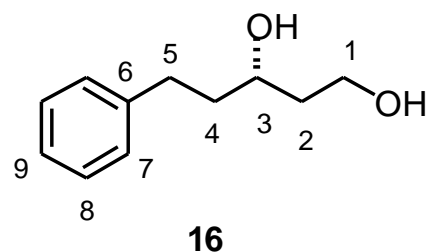

Following General Procedure 4, a solution of $93 \mathrm{mg}$ of (2E,5S)-5-hydroxy-7-phenyl-2heptenoic acid ethyl ester $(2 E, 5 S)$-4ac $(0.37 \mathrm{mmol})$ in $5 \mathrm{~mL}$ of pentane was treated with a solution of ozone in oxygen followed by reduction with $2.55 \mathrm{~mL}$ of a solution of $\mathrm{LiAlH}_{4}$ in THF (0.58 M, $1.48 \mathrm{mmol}, 4$ equiv) to yield, after column chromatography (silica gel, EtOAc/hexanes, 2/1), $42 \mathrm{mg}(63 \%)$ of $(5 S)-16^{10}$ as a colorless oil.

Data for $(3 S)-\mathbf{1 6}$ :

매 NMR: $\quad\left(400 \mathrm{MHz}, \mathrm{CDCl}_{3}\right)$

7.30-7.18 (m, 5 H, HC(7,8,9)), 3.89 (m, 2 H, $\left.\mathrm{H}_{2} \mathrm{C}(1)\right), 3.82$ (m, $\left.1 \mathrm{H}, \mathrm{HC}(3)\right)$, 2.81-

2.65 (m, 2 H, $\left.\mathrm{H}_{2} \mathrm{C}(5)\right), 2.60$ (s, $\left.1 \mathrm{H}, \mathrm{OH}\right), 1.85-1.70$ (m, $4 \mathrm{H}, 2$ x H$\left._{2} \mathrm{C}(2,4)\right)$

${ }^{13} \mathrm{C} \mathrm{NMR}: \quad\left(100 \mathrm{MHz}, \mathrm{CDCl}_{3}\right)$

141.88 (C(6)), $128.43(\mathrm{C}(7)), 128.39$ (C(8)), $125.87(\mathrm{C}(9)), 71.53(\mathrm{C}(3)), 61.72$ (C(1)), $39.30(\mathrm{C}(5)), 38.25(\mathrm{C}(2)), 31.84(\mathrm{C}(4))$

Opt. Rot.: $\quad[\alpha]_{\mathrm{D}}^{24}-10.2(\mathrm{c}=1.32$, EtOH $)$, lit. $[\alpha]_{\mathrm{D}}^{24}-7.21(\mathrm{c}=1.52, \mathrm{EtOH})$ for $(3 S)-\mathbf{1 6}^{10}$

TLC: $\quad R_{f} 0.26($ EtOAc/hexanes, 2/1) [p-anisaldehyde]

(3R)-1-Phenylpropane-1,3-diol ((3R)-14) from (2E,5R)-5-Hydroxy-2-methyl-5-phenyl-2pentenoic Acid Methyl Ester ((2E,5R)-4ba)

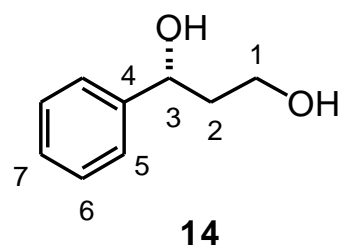

Following General Procedure 4, a solution of $95 \mathrm{mg}$ of (2E,5R)-5-Hydroxy-2-methyl-5phenyl-2-pentenoic acid methyl ester $(2 E, 5 R)-4 \mathbf{b a}(0.43 \mathrm{mmol})$ in $5 \mathrm{~mL}$ of pentane was treated 
with a solution of ozone in oxygen followed by reduction with $2.96 \mathrm{~mL}$ of a solution of $\mathrm{LiAlH}_{4}$ in THF (0.58 M, $1.72 \mathrm{mmol}, 4$ equiv) to yield, after column chromatography (silica gel, EtOAc/hexanes, $2 / 1), 47 \mathrm{mg}(71 \%)$ of $(3 R)-14^{9}$ as a colorless oil.

Data for $(3 R)-14:$

1 NMR: $\quad\left(400 \mathrm{MHz}, \mathrm{CDCl}_{3}\right)$

7.36-7.25 (m, $5 \mathrm{H}, \mathrm{HC}(5,6,7)), 4.96(\mathrm{dd}, J=8.5,3.9,1 \mathrm{H}, \mathrm{HC}(3)), 3.86(\mathrm{t}, J=5.6$, $\left.2 \mathrm{H}, \mathrm{H}_{2} \mathrm{C}(1)\right), 2.55$ (s, $\left.1 \mathrm{H}, \mathrm{OH}\right), 2.1-1.85$ (m, $\left.2 \mathrm{H}, \mathrm{H}_{2} \mathrm{C}(2)\right)$

Opt. Rot.: $\quad[\alpha]_{\mathrm{D}}^{24}+57.1\left(\mathrm{c}=0.978, \mathrm{CHCl}_{3}\right)$, lit. $[\alpha]_{\mathrm{D}}^{24}-63.0\left(\mathrm{c}=0.958, \mathrm{CHCl}_{3}\right)$ for $(3 S)-14{ }^{9}$

\section{(4R)-4-(Acetyloxy)-4-phenyl-2-butanone ((4R)-17) from $(2 E, 5 R)-5$-Hydroxy-3-methyl-5-} phenyl-2-pentenoic Acid Ethyl Ester ((2E,5R)-4ca)

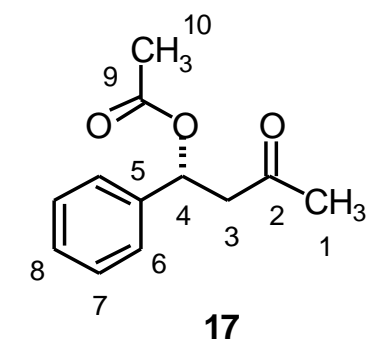

To a $25 \mathrm{~mL}$, 1-neck flask containing a solution of $53 \mathrm{mg}$ of $(2 E, 5 R)-5$-Hydroxy-3methyl-5-phenyl-2-pentenoic acid ethyl ester $(2 E, 5 R)-4 \mathbf{c a}(0.23 \mathrm{mmol})$ in $0.5 \mathrm{~mL}$ of acetic anhydride (5.3 mmol, 23 equiv) was slowly added $0.5 \mathrm{~mL}$ of pyridine ( $6.2 \mathrm{mmol}, 27$ equiv). The

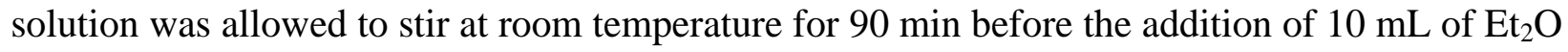
. The cloudy solution was then washed with $0.1 \mathrm{M} \mathrm{HCl}(20 \mathrm{~mL})$ followed by washing with brine $(20 \mathrm{~mL})$. The organic layer was then separated and dried over $\mathrm{Na}_{2} \mathrm{SO}_{4}(5 \mathrm{~g})$, filtered and the filtrate was concentrated in vасио. The residue was then transferred to a $25 \mathrm{~mL}, 1$-neck flask and dissolved in a mixture of $2 \mathrm{~mL} \mathrm{CH}_{2} \mathrm{Cl}_{2}, 2 \mathrm{~mL} \mathrm{CH} \mathrm{CH}_{3} \mathrm{CN}$ and $2 \mathrm{~mL} \mathrm{H}_{2} \mathrm{O}$. To this solution was added $1 \mathrm{mg}$ of $\mathrm{RuCl}_{3}-\mathrm{H}_{2} \mathrm{O}$ (0.0046 mmol, 0.02 equiv) and $206 \mathrm{mg}$ of $\mathrm{NaIO}_{4}$ (0.96 mmol, 4.2 equiv). The biphasic mixture was stirred at room temperature for $2 \mathrm{~h}$ prior to addition of $10 \mathrm{~mL}$ of $0.1 \mathrm{M}$ $\mathrm{HCl}$. The solution was then extracted with $\mathrm{CH}_{2} \mathrm{Cl}_{2}(2 \times 10 \mathrm{~mL})$. The combined organic layers were washed with brine $(10 \mathrm{~mL})$, dried over $\mathrm{Na}_{2} \mathrm{SO}_{4}(5 \mathrm{~g})$, filtered and the filtrate was 
concentrated in vacuo. The residue was purified by column chromatography (silica gel, hexanes/EtOAc, 4/1) to yield $28 \mathrm{mg}(55 \%)$ of $(4 R)-17^{11}$ as a colorless oil.

Data for $(4 R)-17$ :

${ }^{1} \mathrm{H}$ NMR: $\quad\left(400 \mathrm{MHz}, \mathrm{CDCl}_{3}\right)$

7.34-7.29 (m, $5 \mathrm{H}, \mathrm{HC}(6,7,8)), 6.18(\mathrm{dd}, J=8.5,4.9,1 \mathrm{H}, \mathrm{HC}(4)), 3.11$ (dd, $J=$ 16.6, 8.8, $1 \mathrm{H}, \mathrm{HC}(3)), 2.82(\mathrm{dd}, J=16.6,4.9,1 \mathrm{H}, \mathrm{HC}(3)), 2.14$ (s, $\left.3 \mathrm{H}, \mathrm{H}_{3} \mathrm{C}(1)\right)$, 2.03 (s, $\left.3 \mathrm{H}, \mathrm{H}_{3} \mathrm{C}(10)\right)$

${ }^{13} \mathrm{C} \mathrm{NMR}: \quad\left(100 \mathrm{MHz}, \mathrm{CDCl}_{3}\right)$

$204.61(\mathrm{C}(2)), 169.77(\mathrm{C}(9)), 139.62(\mathrm{C}(5)), 128.58$ (C(7)), $128.21(\mathrm{C}(6)), 126.39$ (C(8)), 71.54 (C(4)), $49.80(\mathrm{C}(3)), 30.40$ (C(10)), 21.03 (C(1))

Opt. Rot.: $\quad[\alpha]_{\mathrm{D}}^{24}+60.0\left(\mathrm{c}=0.71, \mathrm{CHCl}_{3}\right)$, lit. $[\alpha]_{\mathrm{D}}^{24}+64.6\left(\mathrm{c}=0.71, \mathrm{CHCl}_{3}\right)$ for $(3 R)-\mathbf{1 7}^{11}$

TLC: $\quad R_{f} 0.35$ (hexanes/EtOAc, 4/1) [UV(254)/KMnO $\left.{ }_{4}\right]$

(2R,3R)-2-Methyl-1-phenylpropane-1,3-diol $((2 R, 3 R)-18)$ from $(2 E, 4 R, 5 R)-5$-Hydroxy-4methyl-5-phenyl-2-pentenoic Acid tert-Butyl Ester ((2E,4R,5R)-4da)

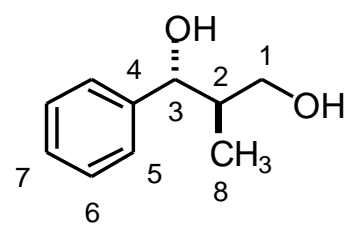

18

Following General Procedure 4, a solution of $95 \mathrm{mg}$ of $(2 E, 4 R, 5 R)$-5-hydroxy-4-methyl5-phenyl-2-pentenoic acid tert-butyl ester $(2 E, 4 R, 5 R)-4 d a(0.36 \mathrm{mmol})$ in $5 \mathrm{~mL}$ of pentane was treated with a solution of ozone in oxygen followed by reduction with $2.48 \mathrm{~mL}$ a solution of $\mathrm{LiAlH}_{4}$ in THF (0.58 M, $1.44 \mathrm{mmol}, 4$ equiv) to yield, after column chromatography (silica gel, EtOAc/hexanes, 2/1), $26 \mathrm{mg}(44 \%)$ of $(2 R, 3 R)-\mathbf{1 8}^{9}$ as a colorless oil.

Data for $(2 R, 3 R)-\mathbf{1 8}$ :

1 NMR: $\quad\left(400 \mathrm{MHz}, \mathrm{CDCl}_{3}\right)$

7.37-7.26 (m, $5 \mathrm{H}, \mathrm{HC}(5,6,7)), 4.50(\mathrm{~d}, J=8.5,1 \mathrm{H}, \mathrm{HC}(3)), 3.70(\mathrm{~m}, 2 \mathrm{H}$, $\left.\mathrm{H}_{2} \mathrm{C}(1)\right), 3.19$ (s, $\left.2 \mathrm{H}, \mathrm{OH}\right), 2.03\left(\mathrm{~m}, 1 \mathrm{H}, \mathrm{H}_{2} \mathrm{C}(2)\right), 0.67$ (d, J= 7.1, $3 \mathrm{H}, \mathrm{H}_{3} \mathrm{C}(8)$ ) 
${ }^{13} \mathrm{C} \mathrm{NMR:} \quad\left(100 \mathrm{MHz}, \mathrm{CDCl}_{3}\right)$

$143.29(\mathrm{C}(4)), 128.39(\mathrm{C}(6)), 127.80(\mathrm{C}(5)), 126.66(\mathrm{C}(7)), 80.83(\mathrm{C}(3)), 67.92$ (C(1)), $41.51(\mathrm{C}(2)), 13.75(\mathrm{C}(8))$

Opt. Rot.: $\quad[\alpha]_{\mathrm{D}}^{24}+35.17\left(\mathrm{c}=0.34, \mathrm{CHCl}_{3}\right)$, lit. $[\alpha]_{\mathrm{D}}^{24}-46.8\left(\mathrm{c}=0.35, \mathrm{CHCl}_{3}\right)$ for $(2 S, 3 S)-\mathbf{1 8} 9$

TLC: $\quad R_{f} 0.47$ (EtOAc/hexanes, 2/1) [p-anisaldehyde]

\section{(2E)-2-Pentenoic Acid tert-Butyl Ester ((2E)-19)}

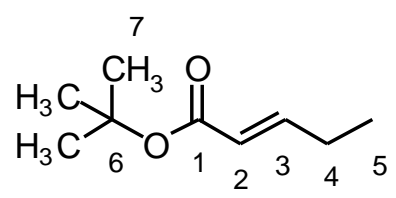

A $250 \mathrm{~mL}$ Parr flask containing a solution of $22.7 \mathrm{~mL}$ of trans-2-pentenoic acid $(0.225$ $\mathrm{mol})$ and $2.25 \mathrm{~mL}$ of conc. sulfuric acid (0.04 mol, 0.17 equiv) in $10 \mathrm{~mL}$ of $\mathrm{Et}_{2} \mathrm{O}$ was cooled to $-78{ }^{\circ} \mathrm{C}$ before condensation of $\sim 30 \mathrm{~mL}$ of isobutylene $(0.34 \mathrm{mmol}, 1.5$ equiv) into the solution. The flask was then placed in a sealed Parr shaker and it was shaken for $24 \mathrm{~h}$. The flask was vented and the excess isobutylene was removed by sparging the solution with an $\mathrm{N}_{2}$ stream. The solution was diluted with $50 \mathrm{~mL}$ of $\mathrm{Et}_{2} \mathrm{O}$, washed with sat. aq. $\mathrm{NaHCO}_{3}$ solution $(50 \mathrm{~mL})$, brine $(50 \mathrm{~mL})$, dried over $\mathrm{K}_{2} \mathrm{CO}_{3}(10 \mathrm{~g})$, filtered and the filtrate was concentrated by distillation of the remaining $\mathrm{Et}_{2} \mathrm{O}$ at atmospheric pressure. The residue was then purified by short path distillation to yield $8.94 \mathrm{~g}(25 \%)$ of $(2 E)-\mathbf{1 9}^{12}$ as a colorless oil.

\section{Data for (2E)-19:}

bp: $\quad 72{ }^{\circ} \mathrm{C}(32 \mathrm{~mm} \mathrm{Hg})$

${ }^{1} \mathrm{H}$ NMR: $\quad\left(400 \mathrm{MHz}, \mathrm{CDCl}_{3}\right)$

$6.90(\mathrm{dt}, J=15.1,6.3,1 \mathrm{H}, \mathrm{HC}(3)), 5.74(\mathrm{dt}, J=15.1,1.7,1 \mathrm{H}, \mathrm{HC}(2)), 2.18$ (dpent, $\left.J=1.4,7.6,2 \mathrm{H}, \mathrm{H}_{2} \mathrm{C}(4)\right), 1.47$ (s, $\left.9 \mathrm{H}, \mathrm{H}_{3} \mathrm{C}(7)\right), 1.04$ (t, $J=7.6,3 \mathrm{H}$, $\left.\mathrm{H}_{3} \mathrm{C}(5)\right)$

${ }^{13} \mathrm{C} \mathrm{NMR}: \quad\left(100 \mathrm{MHz}, \mathrm{CDCl}_{3}\right)$

166.26 (C(1)), 149.38 (C(3)), 122.03 (C(2)), 79.95 (C(6)), 28.12 (C(4)), 25.10 $(\mathrm{C}(7)), 12.19(\mathrm{C}(5))$ 


\section{References:}

1. Denmark, S. E.; Wynn T. J. Am Chem. Soc. 2001, 123, 6199.

2. Denmark, S. E., Wynn, T., Beutner, G. L. J. Am. Chem. Soc. 2002, 124, 13405.

3. Hertler, W. R.; Reddy, G. S.; Sogah, D. Y. J. Org. Chem. 1988, 53, 3532.

4. Hunter, T. J.; O'Doherty, G. A. Org. Lett. 2001, 3, 1049.

5. Sumida, S.; Ohga, M.; Mitani, J.; Nokami, J. J. Am. Chem. Soc. 2000, 122, 1310.

6. Dugger, R. W.; Heathcock, C. H. J. Org. Chem. 1980, 45, 1181.

7. Singer, R. A.; Carriera, E. M. J. Am. Chem. Soc. 1995, 117, 12360.

8. Kobayashi, S.; Ishitani, H.; Yamashita, Y.; Ueno, M.; Shimizu, H. Tetrahedron 2001, 57, 861 .

9. Masamune, S.; Sato, T.; Kim, B.; Wollman, T.A. J. Am. Chem. Soc. 1986, 108, 8279.

10. Nunez, M. T.; Martin, V. S. J. Org. Chem. 1990, 55, 1928.

11. Nair, M. S.; Joly, S. Tetrahedron: Asymmetry 2000, 11, 2049.

12. Hine, J.; Kanagasabapathy, V. M. J. Org. Chem. 1982, 47, 2745. 